October 22, 2018

\title{
Comments on Galilean conformal field theories and their geometric realization
}

\author{
Dario Martelli ${ }^{1}$ and Yuji Tachikawa ${ }^{2}$ \\ ${ }^{1}$ Department of Physics, \\ Swansea University, \\ Singleton Park, Swansea, SA2 8PP, U.K. \\ and \\ 2 School of Natural Sciences, \\ Institute for Advanced Study, \\ Einstein Drive, Princeton, New Jersey, U.S.A.
}

\begin{abstract}
We discuss non-relativistic conformal algebras generalizing the Schrödinger algebra. One instance of these algebras is a conformal, acceleration-extended, Galilei algebra, which arises also as a contraction of the relativistic conformal algebra. In two dimensions, this admits an "exotic" central extension, whereby boosts do not commute. We study general properties of non-relativistic conformal field theories with such symmetry. We realize geometrically the symmetry in terms of a metric invariant under the exotic conformal Galilei algebra, although its signature is neither Lorentzian nor Euclidean. We comment on holographic-type calculations in this background.
\end{abstract}




\section{Contents}

1 Introduction 2

2 Conformal Galilei algebras

2.1 The Schrödinger algebra . . . . . . . . . . . . . . 4

2.2 "Spin-l" conformal Galilei algebras . . . . . . . . . . . 6 6

2.3 Central extensions ........................... 8

3 Exotic conformal Galilei algebra from contraction $\quad 12$

3.1 Improved contraction . . . . . . . . . . . . . . 12

3.2 Contraction of the Dirac equation . . . . . . . . . . . . . 14

4 Properties of CFTs with exotic conformal Galilei symmetry

4.1 Action on operators . . . . . . . . . . . . . . 15

4.2 State-operator correspondence . . . . . . . . . . . . 16

4.3 Unitarity bound . . . . . . . . . . . . . . . . 17

4.4 Two-point functions ......................... 18

5 Field theories with exotic conformal Galilei symmetry 20

5.1 Free field action . . . . . . . . . . . . . . . 20

5.2 Chern-Simons-matter action . . . . . . . . . . . . . 22

5.3 Free field wave function . . . . . . . . . . . . . . . 23

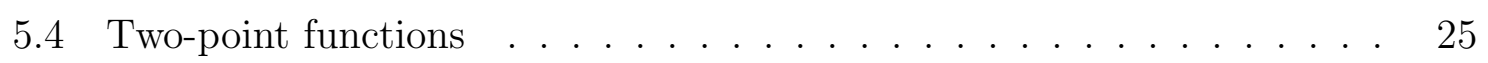

6 Geometric realization 25

6.1 Invariant metric . . . . . . . . . . . . . . . . 25

6.2 "Holographic" two-point function . . . . . . . . . . . . . 26

\begin{tabular}{ll|l|}
7 & Conclusions & 29 \\
\hline
\end{tabular}

A Mechanics with exotic conformal Galilei symmetry 31 


\section{Introduction}

Recently there has been considerable activity in extending the Anti-de Sitter/conformal field theory (AdS/CFT) correspondence to various low-energy physical systems. In many instances these systems are non-relativistic, implying that their fundamental symmetry is Galilean. One example of Galilean symmetry is the Schrödinger symmetry, which is the symmetry of the free Schrödinger equation. It was proposed in [1, 2] that some gravity backgrounds with Schrödinger symmetry should be holographically dual to certain conformal quantum mechanics relevant for the description of cold atoms [3].

In [4, 5, 6] it was shown that the backgrounds of [1, 2] arise as solutions of String Theory. One way to embed these geometries in String Theory is based on the fact that the field theories arise from the discrete light cone quantization (DLCQ) of relativistic conformal field theories. At the level of the symmetry, it utilizes the fact that the Schrödinger group is a subgroup of the relativistic conformal group [7, 8, 9]. On the gravity side this corresponds to the light-like compactification of $\mathrm{AdS}_{5}$, or more generally to a "twisted" version of this, obtained by a chain of String dualities. On the field theory side, it corresponds to (the DLCQ of) certain dipole deformations of the parent relativistic CFT [10]. Subtleties involved in these constructions were discussed in [4].

A natural question to ask is whether there exist non-relativistic conformal field theories different from those enjoying Schrödinger symmetry, that may be realized holographically as in [1, 2]. There is an abundance of symmetry groups, comprising the Galilei group as a subgroup 1 , and some of them are known to be symmetries of certain condensed matter problems. If one in addition demands conformal invariance, it turns out that there is a class of conformal Galilei algebras, generalizing precisely the Schrödinger algebra [12, 13]. The non-relativistic Navier-Stokes equations are an example where these symmetries are relevant [15, 16]. For more examples in condensed matter see [17] and references therein.

An interesting conformal Galilei algebra, which will be the main focus of the paper, is closely related to the Schrödinger one. The main differences with respect to the latter are that dilatations act as in the relativistic case, $D:(t, \vec{x}) \rightarrow(\lambda t, \lambda \vec{x})$, and it contains new generators corresponding to constant accelerations [18, 19]. Furthermore, this algebra does not admit the usual Galilean central extension by the mass operator $M$. It thus necessarily describes massless particles. On the other hand, in $d=2$

\footnotetext{
${ }^{1}$ Note that the Lifshitz group considered in [1] is not of this type.
} 
spatial dimensions, it admits a different central extension, by an operator we denote $\Theta$. Following the literature, we will refer to this centrally extended conformal Galilei algebra as "exotic" [14. One motivation for studying the centrally extended algebra is that the central extension played a key role in the geometric realization of the Schrödinger symmetry, through the Bargmann construction [8, 9, 20]. This is related to the fact that in non-relativistic quantum mechanics wave functions transform "up to a phase" [21] under the symmetry group, while they form true representations for the centrally extended group. Then one would expect that adding an extra direction, conjugate to $\Theta$, the exotic conformal Galilei algebra should be realized geometrically. In fact, this is roughly correct, as we will show.

Another intriguing feature of this algebra is that it arises as a non-relativistic contraction [22] of the conformal algebra [23, 24, 25, 26]. The contraction amounts to taking a certain scaling limit of the generators. If these are Killing vectors of a metric, as in AdS/CFT, then the contraction can be implemented in the metric. Indeed this is what happens in the Penrose limit: the contraction of the metric in this case gives generically pp-wave space-times, while in the field theory side it corresponds to the scaling limit discussed in [27]. In the context of non-relativistic AdS/CFT, an analogous scaling limit was performed in [4]. The String Theory side of this limit had been considered befor 2 in $[28$. However, the situation here is different: a scaling limit of the AdS metric does not give rise to a non-degenerate bulk metric realizing the symmetry. We instead perform a modified version of the contraction involving spin generators, and we show that when applied to the Dirac equation this produces non-trivial results in the limit. In particular, the centrally-extended algebra, and a free field wave equation, replacing the Schrödinger equation, can be derived in this way. The interpretation of the exotic central extension as a non-relativistic limit of spin was first pointed out in [23, 24, 25].

We will study some generic features of conformal field theories with exotic Galilean symmetry. As examples, we will present a free field and a Chern-Simons-matter-type action, invariant under the symmetry group, at least classically. Following the strategy of [8, 9, 20] and of [1, 2], we will present a metric which realizes the exotic conformal Galilei group as its isometry group. Disappointingly, however, this metric has signature $(3,4)$, and it turns out to be a "wrong-signature" versions of $\mathrm{AdS}_{7}$, or its deformation preserving the Schrödinger group. Therefore its use for AdS/CFT applications appears problematic. Despite this severe issue, we point out that a blind application of the

\footnotetext{
${ }^{2}$ We thank the authors of [28 for bringing their work to our attention.
} 
AdS/CFT prescriptions yields a consistent result for the two-point functions.

The rest of the paper is organized as follows. In Sec. 2 we briefly review the Schrödinger algebra and other conformal Galilei algebras. We then discuss the central extensions of these algebras, and of the $l=1$ case in more detail. Sec. 3 discusses the origin of the algebra as a non-relativistic contraction. In Sec. 4 we study formal properties of field theories with exotic conformal Galilei algebras. In Sec. 5 we discuss explicit field theories examples. In Sec. 6] we present an invariant metric and comment on a holographic-type computation of two-point functions, based on this background metric. In Sec. 7 we conclude. In Appendix A we discuss a "particle" Lagrangian with exotic conformal Galilei symmetry and its quantization.

Note: while writing the present article, a paper discussing conformal Galilei algebras appeared [30. Just before submitting, we became aware of two other related papers [31], [32]. However, our main results have not been discussed in these references.

\section{Conformal Galilei algebras}

\subsection{The Schrödinger algebra}

We will begin by recalling the Schrödinger group and its geometric realization. As we will see, the conformal Galilei groups that we will discuss in this paper are a natural generalization of the Schrödinger one. The fundamental symmetry of a non-relativistic system is the Galilei group. A point in $d$-dimensional Euclidean space $\vec{x}$, at a given time $t$, transforms under the Galilei group as

$$
t \rightarrow t+e, \quad \vec{x} \rightarrow R \vec{x}+\vec{b} t+\vec{c}
$$

where $R \in S O(d), \vec{b}, \vec{c} \in \mathbb{R}^{d}, e \in \mathbb{R}$. Let us denote by $X$ the pair $(t, \vec{x})$, and denote by $P(\vec{c})$ and $K(\vec{b})$ the geometric actions of finite translation by $\vec{c}$ and finite boost by $\vec{b}$, respectively.

Quantum mechanically, wave functions must form a projective unitary representation of the group, which is equivalent to a unitary representation of the centrally extended Bargmann group [21]. Consider the free Schrödinger equation with mass $m$,

$$
\left(i \partial_{t}+\frac{1}{2 m} \partial_{i} \partial_{i}\right) \psi=0
$$

One can trivially promote the geometric action of the translation $P(\vec{c})$ to an action 
$U_{P(\vec{c})}$ on the wavefunction by defining

$$
U_{P(\vec{c})} \psi(X)=\psi(P(-\vec{c}) X)
$$

however the action $\psi(X) \rightarrow \psi(K(-\vec{b}) X)$ does not map a solution to another solution. Defining the unitary operator $U_{K(\vec{b})}$ by further multiplying by a phase which depends both on space and time,

$$
U_{K(\vec{b})} \psi(X)=\exp \left[-i m \vec{b} \cdot \vec{x}+\frac{i}{2} m t \vec{b}^{2}\right] \psi(K(-\vec{b}) X),
$$

one finds that the plane wave solution $\psi_{E, \vec{p}}=\exp (i E t-i \vec{p} \cdot \vec{x})$ is mapped to another plane wave,

$$
\psi_{E, \vec{p}} \rightarrow U_{K(\vec{b})} \psi_{E, \vec{p}}=\psi_{E^{\prime}, \vec{p}^{\prime}}
$$

where

$$
E^{\prime}=\frac{(\vec{p}+m \vec{b})^{2}}{2 m}, \quad \vec{p}^{\prime}=\vec{p}+m \vec{b}
$$

The operators $U_{P(\vec{c})}$ and $U_{K(\vec{b})}$ no longer commute, instead we have

$$
U_{P(\vec{c})} U_{K(\vec{b})}=\exp (i m \vec{b} \cdot \vec{c}) U_{K(\vec{b})} U_{P(\vec{c})}
$$

i.e. they realize a central extension. One can add an extra coordinate $\xi$ and consider the wave function $\psi=\exp (i m \xi+i E t-i \vec{p} \cdot \vec{x})$. This is invariant, provided $\xi$ transforms as

$$
\xi \rightarrow \xi+\vec{b} \cdot \vec{x}+\frac{1}{2} t \vec{b}^{2}
$$

In turn, the Schrödinger equation may be interpreted as the Klein-Gordon equation in the Bargmann space $\mathbb{R}^{d+1,1}$, with metric

$$
\mathrm{d} s^{2}=2 \mathrm{~d} \xi \mathrm{d} t+(\mathrm{d} \vec{x})^{2}
$$

In fact, the symmetry group of the Schrödinger equation is not just the Bargmann group, but it comprises also dilatations, $D:(t, \vec{x}) \rightarrow\left(\lambda^{2} t, \lambda \vec{x}\right)$, and special conformal transformations, $C:(t, \vec{x}) \rightarrow(1+f t)^{-1}(t, \vec{x})$. The complete action of the Schrödinger group on the extended space-time is

$$
\begin{aligned}
& \vec{x} \rightarrow \frac{R \vec{x}+\vec{b} t+\vec{c}}{f t+g}, \quad t \rightarrow \frac{d t+e}{f t+g}, \\
& \xi \rightarrow \xi+\frac{f}{2} \frac{(R \vec{x}+\vec{b} t+\vec{c})^{2}}{f t+g}-\vec{b} \cdot R \vec{x}-\frac{t}{2} \vec{b}^{2}+h
\end{aligned}
$$


where $d g-e f=1$. The corresponding algebra 3 of generators is given by

$$
\begin{array}{lll}
{[D, H]=H,} & {[D, C]=-C,} & {[C, H]=2 D,} \\
{\left[H, P_{i}\right]=0,} & {\left[D, P_{i}\right]=\frac{1}{2} P_{i},} & {\left[C, P_{i}\right]=K_{i},} \\
{\left[H, K_{i}\right]=-P_{i},} & {\left[D, K_{i}\right]=-\frac{1}{2} K_{i},} & {\left[C, K_{i}\right]=0}
\end{array}
$$

plus the central extension

$$
\left[P_{i}, K_{j}\right]=\delta_{i j} M
$$

Momenta $P_{i}$ and boosts $K_{i}$ transform as vectors under spatial rotations $J_{i j}$, and as a doublet of the $S L(2 ; \mathbb{R})$ sub-algebra generated by $H, D, C$. Based on the above remarks, it can be shown that the Schrödinger algebra is a sub-algebra of the relativistic conformal algebra in one higher space-time dimension [7]. This was indeed a key observation for the holographic realization of the Schrödinger symmetry [1, 2].

\section{2 "Spin-l" conformal Galilei algebras}

We now present a class of conformal extensions of the Galilei algebra labeled by a half-integer $l$, discussed in [12, 13]. The smallest instance $l=1 / 2$ corresponds to the (non centrally-extended) Schrödinger algebra. The next case, $l=1$, is an extension of the Galilei group comprising constant accelerations, and we will study it in more detail. Below, we follow [12, 13] and present the algebras without central extensions. Those with central extensions will be discussed in the next subsection.

The action on space-time may be written as

$$
\vec{x} \rightarrow \frac{R \vec{x}+t^{2 l} \overrightarrow{c_{2 l}}+\cdots+t^{2} \overrightarrow{c_{2}}+t \overrightarrow{c_{1}}+\overrightarrow{c_{0}}}{(f t+g)^{2 l}}, \quad t \rightarrow \frac{d t+e}{f t+g}
$$

where $R \in S O(d), \vec{c}_{n} \in \mathbb{R}^{d}$ and $d g-e f=1$, generalizing the $l=1 / 2$ transformations (2.10). From this we see that the infinitesimal action is generated by the following vector fields

$$
\begin{array}{rlrl}
H & =\partial_{t}, & D & =-t \partial_{t}-l x_{i} \partial_{i}, \quad C=t^{2} \partial_{t}+2 l t x_{i} \partial_{i}, \\
J_{i j} & =-\left(x_{i} \partial_{j}-x_{j} \partial_{i}\right), \quad P_{i}^{n}=(-t)^{n} \partial_{i},
\end{array}
$$

where $i=1, \ldots, d$ and $n=0, \ldots, 2 l$. Notice that the dilatations act as

$$
t \rightarrow \lambda t, \quad \vec{x} \rightarrow \lambda^{l} \vec{x}
$$

\footnotetext{
${ }^{3}$ The normalization of the $D$ here is $1 / 2$ of the usual normalization in the Schrödinger algebra.
} 
corresponding to a dynamical critical exponent $z=1 / l \leq 2$.

The algebra satisfied by the generators $\left\{H, D, C, J_{i j}, P_{i}^{n}\right\},(n=0, \ldots, 2 l ; i=1, \ldots, d)$ comprises the following non-zero commutation relations

$$
\begin{aligned}
& {[D, H]=H, \quad[D, C]=-C, \quad[C, H]=2 D,} \\
& {\left[H, P_{i}^{n}\right]=-n P_{i}^{n-1}, \quad\left[D, P_{i}^{n}\right]=(l-n) P_{i}^{n}, \quad\left[C, P_{i}^{n}\right]=(2 l-n) P_{i}^{n+1},} \\
& {\left[J_{i j}, P_{k}^{n}\right]=\delta_{i k} P_{j}^{n}-\delta_{j k} P_{i}^{n}, \quad\left[J_{i j}, J_{k l}\right]=\delta_{i k} J_{j l}+\delta_{j l} J_{i k}-\delta_{i l} J_{j k}-\delta_{j k} J_{i l} \quad .}
\end{aligned}
$$

We can then take this as an abstract definition of the algebra, independent of the specific realization (2.14). Notice that thes generators are anti-Hermitean, and $H, D, C$ form an $S L(2 ; \mathbb{R})$ sub-algebra, familiar from the Schrödinger algebra. The generators $P_{i}^{n}$ transform in the spin- $l$ representation of this $S L(2 ; \mathbb{R})$, and as vectors of the $S O(d)$ sub-algebra of spatial rotations generated by $J_{i j}$ in the usual way.

Setting $l=1 / 2$ and defining $P_{i}^{0}=P_{i}, P_{i}^{1}=K_{i}$, we recover the Schrödinger algebra (2.11), albeit without central extension. For $l=1$, there is one extra set of generators, transforming as a vector of $S O(d)$, that we will denote $P_{i}^{2}=F_{i}$. It generates accelerations $\vec{x} \rightarrow \vec{x}+t^{2} \vec{a}$. For future reference, let us write down the non-trivial commutators:

$$
\begin{array}{lll}
{\left[H, P_{i}\right]=0,} & {\left[D, P_{i}\right]=P_{i},} & {\left[C, P_{i}\right]=2 K_{i},} \\
{\left[H, K_{i}\right]=-P_{i},} & {\left[D, K_{i}\right]=0,} & {\left[C, K_{i}\right]=F_{i},} \\
{\left[H, F_{i}\right]=-2 K_{i},} & {\left[D, F_{i}\right]=-F_{i},} & {\left[C, F_{i}\right]=0 .}
\end{array}
$$

Notice that the dynamical exponent in this case is $z=1$, the same as the relativistic one. One interesting feature of this algebrat is that it can be obtained as a nonrelativistic contraction of the relativistic conformal algebra [26]. We will discuss this aspect later in Sec. 3 .

\section{Infinite dimensional extension}

It is known that the Schrödinger algebra admits an infinite dimensional Virasoro-like extension [33, 34], which should be relevant for holographic applications [35]. We notice that such Virasoro-like extensions exist for every $l$. This is easily showed in the representation (2.14). First, we rename the $S L(2 ; \mathbb{R})$ generators as $L^{-1}=-H$,

\footnotetext{
${ }^{4}$ In fact also the centrally-extended algebra, to be discussed momentarily.
} 
$L^{0}=D$, and $L^{1}=-C$, and then we define the following operators

$$
\begin{aligned}
L^{n} & =-t^{n+1} \partial_{t}-l(n+1) t^{n} x_{i} \partial_{i}, \\
P_{i}^{n} & =-t^{n+l} \partial_{i}, \\
J_{i j}^{n} & =-t^{n}\left(x_{i} \partial_{j}-x_{j} \partial_{i}\right)
\end{aligned}
$$

where $n \in \mathbb{Z}$ for the $L^{n}$, and $n \in \mathbb{Z}+l$ for the $P_{i}^{n}$. The generators $\left\{P_{i}^{-l}, \ldots, P_{i}^{l}\right\}$ are those of the finite-dimensional conformal Galilei algebras (after a relabeling $n \rightarrow n-l$ and multiplying by \pm 1 ). The commutators are then written in the compact form

$$
\begin{aligned}
& {\left[L^{m}, L^{n}\right]=(m-n) L^{m+n},} \\
& {\left[L^{m}, J_{i j}^{n}\right]=-n J_{i j}^{n},} \\
& {\left[J_{i j}^{m}, J_{k l}^{n}\right]=\delta_{i k} J_{j l}^{m+n}+\delta_{j l} J_{i k}^{m+n}-\delta_{i l} J_{j k}^{m+n}-\delta_{j k} J_{i l}^{m+n},} \\
& {\left[L^{m}, P_{i}^{n}\right]=(l m-n) P_{i}^{m+n},} \\
& {\left[J_{i j}^{m}, P_{k}^{n}\right]=\delta_{i k} P_{j}^{m+n}-\delta_{j k} P_{i}^{m+n} .}
\end{aligned}
$$

The $L^{n}$ generators form a Virasoro algebra, $J_{i j}^{n}$ is an $s o(d)$ current algebra, and $P_{i}^{n}$ give weight- $(l+1)$ Virasoro primaries which are also primary under the current algebra; they do not have any central extension at all at this stage. One can add the usual central extension $\delta_{n+m, 0}\left(n^{3}-n\right) c / 12$ to the $\left[L^{m}, L^{n}\right]$ commutators, which might arise quantum mechanically, or classically as in [36] if we have a holographic dual.

\subsection{Central extensions}

As we reviewed, the Galilei group admits the central extension (2.12), which was originally found by Bargmann [21]. This central extension plays a crucial role in realizing geometrically [8] and holographically [1, 2] the Schrödinger symmetry. Motivated by this, we will now show that there exists a central extension of the spin- $l$ conformal Galilei groups. The algebras in the previous sub-section are of the form $\mathfrak{g} \oplus V$, where $\mathfrak{g}$ is a Lie algebra and $V$ its representation. In our case $\mathfrak{g}$ is $s l(2 ; \mathbb{R}) \oplus s o(d)$ and $V$ is the tensor product of the spin- $l$ representation of $S L(2 ; \mathbb{R})$ and the vector representation of $S O(d)$. The commutator of $g \in \mathfrak{g}$ and $v \in V$ is defined by

$$
[g, v]=g v
$$

where the right hand side stands for the action of $g$ on $v$. For $v, w \in V$ we have

$$
[v, w]=0 .
$$


Since $\mathfrak{g}$ is semisimple, the central extension can only enter in (2.21) as

$$
[v, w]=K(v, w)
$$

where $K(v, w)$ is an antisymmetric pairing. The Jacobi identity is then satisfied if and only if $K(v, w)$ is invariant under $\mathfrak{g}$. Now, the invariant tensor $I^{m n}$ of the spin- $l$ representation of $S L(2 ; \mathbb{R})$ is symmetric or antisymmetric depending on the parity of $2 l$, whereas $S O(d)$ has an antisymmetric invariant tensor $\epsilon_{i j}$ for $d=2$ and a symmetric tensor $\delta_{i j}$ for any $d$.

From these considerations, we conclude that there are two types of central extension of conformal Galilei groups. One exists for any $d$ and half-integral $l$ :

$$
\left[P_{i}^{m}, P_{j}^{n}\right]=I^{m n} \delta_{i j} M
$$

and another which exists only for $d=2$ and integral $l$ :

$$
\left[P_{i}^{m}, P_{j}^{n}\right]=I^{m n} \epsilon_{i j} \Theta
$$

Let us discuss the two cases which we are most interested in. For arbitrary $d$ with $l=1 / 2$, one has the massive central extension (2.12) of the algebra in (2.11). This is the familiar Schrödinger symmetry with the Bargmann extension. In order to realize the centrally extended algebra by the action of vector fields, one needs to add one extra direction $\xi$ which is conjugate to $M$. Equivalently, one can Fourier-transform with respect to the mass $M$. The generators take the form

$$
\begin{array}{ll}
H=\partial_{t}, & D=-\frac{1}{2} x_{i} \partial_{i}-t \partial_{t}, \quad C=t x_{i} \partial_{i}+t^{2} \partial_{t}-\frac{1}{2} x_{i} x_{i} \partial_{\xi}, \\
P_{i}=\partial_{i}, & K_{i}=-t \partial_{i}-x_{i} \partial_{\xi}, \quad J_{i j}=-\left(x_{i} \partial_{j}-x_{j} \partial_{i}\right),
\end{array}
$$

and $M=\partial_{\xi}$.

Next consider $d=2$ with $l=1$. The centrally extended algebra has commutators

$$
\left[K_{i}, K_{j}\right]=\Theta \epsilon_{i j}, \quad\left[P_{i}, F_{j}\right]=-2 \Theta \epsilon_{i j}
$$

in addition to those in (2.17). We denote the eigenvalue of $\Theta$ by $i \theta$. Following [14, 26], we will refer to the resulting symmetry algebra as the exotic conformal Galilei algebra which will be the focus of the rest of our paper. The fact that the two-dimensional Galilei group admits a second central extension $\Theta$, in addition to the mass parameter $M$ has long been known [37, 38, 39], and various systems have been discussed in the literature [40, 18, 41, 19]. We see that the existence of the extra conformal generators only allows $\Theta$, not $M$, as noted in [26]. 
We can represent this algebra by modifying the operators in (2.14) with extra terms which produce the central extension. Concretely, we have

$$
\begin{array}{lll}
H=\partial_{t}, & D=-x_{i} \partial_{i}-t \partial_{t}, & C=2 t x_{i} \partial_{i}+t^{2} \partial_{t}-2 x_{i} \chi_{i}, \\
P_{i}=\partial_{i}, & K_{i}=-t \partial_{i}+\chi_{i} & F_{i}=t^{2} \partial_{i}-2 t \chi_{i}+2 x_{j} \epsilon_{i j} \Theta,
\end{array}
$$

where we require that the operators $\chi_{1,2}$ and $\Theta$ satisfy the Heisenberg commutation relations

$$
\left[\chi_{i}, \chi_{j}\right]=i \Theta \epsilon_{i j}, \quad\left[\chi_{i}, \Theta\right]=0,
$$

and that they commute with the operators defined in (2.14). The action of the modified generators can be seen as the sum of the geometric action (2.14) of the group without the central extension, modified by the part proportional to $\chi_{i}$ and $\Theta$.

Let us now discuss the rotation generator. First of all, notice that in order for the generators $P_{i}, K_{i}, F_{i}$ to transform as vectors under rotations, $\chi_{i}$ should transform as vectors. Then the rotation generator comprises two pieces:

$$
J_{i j}=-\left(x_{i} \partial_{j}-x_{j} \partial_{i}\right)+S_{i j}
$$

where $S_{i j}$ rotates the $\chi_{i}$. Inspired by the observation in [19], we define the rotation generator as $2 i \theta J \equiv F_{i} P_{i}-K_{i} K_{i}$, so that

$$
J=-\epsilon_{i j} x_{i} \frac{\partial}{\partial x_{j}}-\frac{1}{2 i \theta} \chi_{i} \chi_{i}
$$

With this definition we have

$$
\left[J, x_{i}\right]=\epsilon_{i j} x_{j}, \quad\left[J, \chi_{i}\right]=\epsilon_{i j} \chi_{j}
$$

thus this $J$ correctly transforms all of $P_{i}, K_{i}$ and $F_{i}$ as vectors. It is clear that we can think of $\chi_{i}$ as an intrinsic part of the Galilei boost, just as the rotation generator acting on a field with non-trivial spin is not simply $x_{[i} \partial_{j]}$ but $x_{[i} \partial_{j]}+\Sigma_{i j}$. We will pursue this analogy further in Sec. 3. this form naturally arises by taking the contraction of Lorentz generators acting on spinor fields.

We can obtain a realization of the algebra in terms of vector fields, by representing the Heisenberg algebra in an auxiliary three-dimensional space with coordinates $v_{1}, v_{2}$, and $\xi$. In particular, we can take

$$
\Theta=\partial_{\xi}, \quad \chi_{i}=\frac{\partial}{\partial v_{i}}-\frac{1}{2} \epsilon_{i j} v_{j} \partial_{\xi} .
$$


This is a generalization of the Bargmann space to the present case. We will study natural metrics on this space later. The algebra of vector fields is obtained by just plugging (2.32) into (2.27). For the rotation generator, we have

$$
J=-\epsilon_{i j}\left(x_{i} \frac{\partial}{\partial x_{j}}+\frac{1}{2} v_{i} \frac{\partial}{\partial v_{j}}\right)-\frac{1}{2 i \theta} \frac{\partial}{\partial v_{i}} \frac{\partial}{\partial v_{i}}-\frac{i \theta}{8} v_{i} v_{i}
$$

Notice that this does not rotate independently $\partial / \partial v_{i}$ and $v_{i}$. Actually, in this representation the naive definition of the internal rotation generator

$$
S_{i j}=-\left(v_{i} \frac{\partial}{\partial v_{j}}-v_{j} \frac{\partial}{\partial v_{i}}\right)
$$

gives the correct commutation relations, thus both choices are possible in this case. We will see however that the more general definition (2.30) arises naturally from the contraction procedure. Notice that the internal part of the rotation operator (2.30) is a harmonic oscillator Hamiltonian.

The induced infinitesimal action on the extended six-dimensional space with coordinates $\left(t, x_{i}, \xi, v_{i}\right)$ is easily obtained from the vector fields, and reads

$$
\begin{aligned}
& H: t \rightarrow t+e, \\
& D: t \rightarrow t-\lambda t, \quad x_{i} \rightarrow x_{i}-\lambda x_{i}, \\
& C: t \rightarrow t+a t^{2}, \quad x_{i} \rightarrow x_{i}+2 a t x_{i}, \\
& \quad v_{i} \rightarrow v_{i}-2 a x_{i}, \quad \xi \rightarrow \xi+a \epsilon_{i j} x_{i} v_{j}, \\
& P_{i}: x_{i} \rightarrow x_{i}+c_{i}, \\
& K_{i}: x_{i} \rightarrow x_{i}-b_{i} t, \quad v_{i} \rightarrow v_{i}+b_{i}, \quad \xi \rightarrow \xi-\frac{1}{2} \epsilon_{i j} b_{i} v_{j}, \\
& F_{i}: x_{i} \rightarrow x_{i}+a_{i} t^{2}, \quad v_{i} \rightarrow v_{i}-2 a_{i} t, \quad \xi \rightarrow \xi+\epsilon_{i j} a_{i}\left(2 x_{j}+t v_{j}\right), \\
& \Theta: \xi \rightarrow \xi+h .
\end{aligned}
$$

Notice the transformations of the $v_{i}$ may be interpreted as (minus) the transformations of velocities $\dot{x}_{i}$. A general transformation of $\xi$,

$$
\xi \rightarrow \xi+a \epsilon_{i j} x_{i} v_{j}+\epsilon_{i j} a_{i}\left(2 x_{j}+t v_{j}\right)-\frac{1}{2} \epsilon_{i j} b_{i} v_{j}+h
$$

at first sight does not have an obvious interpretation. However it has a natural interpretation in terms quasi-invariance of a free particle mechanical system that we discuss in Appendix $\mathrm{A}$. 


\section{Exotic conformal Galilei algebra from contrac- tion}

One interesting aspect of the $l=1$ conformal Galilei algebra is that it can be obtained as the contraction of the conformal algebra $S O(d+1,2)$, just as the Galilei algebra arises as the contraction of the Poincaré algebra [22]. This raises the hope that applying the contraction to the $\mathrm{AdS}_{d+2}$ metric, we could obtain a non-trivial metric with desired isometries. However, it is easy to see that such hope cannot be realized, as there is no $d+2$ dimensional metric which is non-degenerate with such isometries. The reasoning for this is analogous to that showing that there is no $d+1$ dimensional metric, with ordinary Galilei symmetries. Indeed, performing the limit in the metric gives a degenerate result.

\subsection{Improved contraction}

In this subsection we will discuss a modified contraction that leads to the centrally extended conformal Galilei algebra in $d=2$ dimensions. The role of spin in the contraction was emphasized in [23, 24, 25], however our treatment here is slightly different, in particular we show that the Heisenberg algebra emerges in the limit 5 . We start from an explicit representation of the relativistic conformal algebra, where the generators of $S O(3,2)$ act on operators with spin, and perform a simultaneous contraction of the space-time and spin parts. Namely, we take

$$
\begin{aligned}
\tilde{M}_{\mu \nu} & =x_{\mu} \partial_{\nu}-x_{\nu} \partial_{\mu}+\Sigma_{\mu \nu}, & \tilde{P}_{\mu} & =\partial_{\mu}, \\
\tilde{K}_{\mu} & =x^{\nu} x_{\nu} \partial_{\mu}-2 x_{\mu} x^{\nu} \partial_{\nu}-2 x^{\nu} \Sigma_{\mu \nu}, & \tilde{D} & =x^{\nu} \partial_{\nu},
\end{aligned}
$$

where the $\Sigma_{\mu \nu}$ can be thought of as a finite dimensional representation of $S O(1,2)$. We then set $x_{0}=c t$ and after rescaling the spin generators as

$$
\Sigma_{i 0} \rightarrow c \Sigma_{i 0}, \quad \Sigma_{i j} \rightarrow c^{2} \Sigma_{i j}
$$

we define the following generators

$$
\begin{array}{lll}
P_{i}=\tilde{P}_{i}, & K_{i}=\frac{\tilde{M}_{i 0}}{c}, & F_{i}=\frac{\tilde{K}_{i}}{c^{2}}, \\
H=\tilde{P}_{0}, & D=\tilde{D}, & C=\frac{\tilde{K}_{0}}{c},
\end{array}
$$

\footnotetext{
${ }^{5}$ We have recently learned that this contraction was considered previously in [29], albeit the discussion there concerns a non-conformal algebra, with two central extensions $\Theta$ and $M$.
} 
as well as

$$
J=-\tilde{M}_{12}+c^{2} \Sigma_{12}
$$

Taking the $c \rightarrow \infty$ limit we find the following operators

$$
\begin{array}{lll}
H=\partial_{t}, & D=-t \partial_{t}-x_{i} \frac{\partial}{\partial x_{i}}, & C=t^{2} \partial_{t}+2 t x_{i} \frac{\partial}{\partial x_{i}}+2 x_{i} \Sigma_{i 0}, \\
P_{i}=\frac{\partial}{\partial x_{i}}, & K_{i}=-t \frac{\partial}{\partial x_{i}}-\Sigma_{i 0}, & F_{i}=t^{2} \frac{\partial}{\partial x_{i}}+2 t \Sigma_{i 0}+2 x_{j} \Sigma_{i j}
\end{array}
$$

and $\Theta_{i j}=\Sigma_{i j}$. We will address rotations momentarily. The scaling limit of the commutation relations among the $\Sigma_{\mu \nu}$ gives rise to

$$
\left[\Sigma_{0 i}, \Sigma_{0 j}\right]=\Sigma_{i j}, \quad\left[\Sigma_{i j}, \Sigma_{0 k}\right]=0
$$

Defining $\chi_{i}=\Sigma_{0 i}, \Sigma_{i j}=\epsilon_{i j} \Theta$, we see that these three operators obey precisely the Heisenberg commutation relations (2.28). Indeed it is well known that the Heisenberg group is a contraction of the $S U(2)$ group 6 . One can pick a representation of the Heisenberg algebra, where the $\chi_{i}$ take a concrete form. For example, one could consider a finite dimensional representation in terms of $3 \times 3$ matrices, but this is not unitary. An unitary representation is of course given by $\chi_{1}=s, \chi_{2}=i \theta \partial_{s}$. As we discussed in Sec. 2.3, a unitary representation in terms of vector fields is obtained introducing three auxiliary coordinates $\xi, v_{1}, v_{2}$ and defining (2.32).

Let us now address the rotations. Firstly, notice that with the definition (3.4) one result of the $c \rightarrow \infty$ limit of the commutation relations of the Lorentz rotations $\left[\tilde{M}_{\mu \nu}, \tilde{M}_{\mu \nu}\right]$ is that

$$
\left[J_{i j}, J_{k 0}+\Sigma_{k 0}\right]=\delta_{i k}\left(J_{j 0}+\Sigma_{j 0}\right)-\delta_{j k}\left(J_{i 0}+\Sigma_{i 0}\right) .
$$

While one can check that all other components give a limit which is consistent with the contraction of the $S O(1,2)$ algebra to the Heisenberg one, we see that (3.7) is problematic. In particular, the spatial rotations $J_{i j}$ do not act on $\Sigma_{0 k}$. Thus, the naive contraction procedure is not consistent.

To obtain a consistent contraction we apply the following trick. We consider a trivial central extension of the relativistic algebra, obtained adding a constant to the relativistic spatial rotations:

$$
-\epsilon_{i j} x_{i} \partial_{j} \rightarrow-\epsilon_{i j} x_{i} \partial_{j}+\alpha\left(2 \Sigma_{0 i} \Sigma_{0 i}-\Sigma_{i j} \Sigma_{i j}\right)+\beta
$$

\footnotetext{
${ }^{6}$ We could also consider a different contraction, giving the Euclidean group, but this turns out to be not consistent.
} 
Then the contraction limit is consistent, provided we take $\alpha=i /\left(4 \theta c^{2}\right)$ and $\beta=$ $-i \theta c^{2} / 4$. The rotation generator in the limit is indeed (2.30). In particular, the divergent term $c^{2} \theta$ that needs to be subtracted to get a finite answer in the limit, is analogous to the divergent rest mass $c^{2} M$. We see that the parameter $\theta$ is a remnant of spin in the non-relativistic limit.

\subsection{Contraction of the Dirac equation}

The relevance of spin in the non-relativistic limit discussed in the previous subsection suggests that one should be able to take an appropriate limit of a relativistic equation for fields with spin, for example the Dirac equation. Notice in particular that the non-relativistic limit of the Klein-Gordon equation (in flat space) is degenerate.

Let us then start from the massless Dirac equation in $2+1$ dimensions:

$$
\Gamma^{\mu} \partial_{\mu} \Psi=\left(-\Gamma^{0} \partial_{t}+\Gamma^{1} \partial_{1}+\Gamma^{2} \partial_{2}\right) \Psi=0
$$

where $\Gamma^{a}$ are gamma-matrices of $S O(1,2)$. To take the limit, it is convenient to multiply by $\Gamma^{012}$ :

$$
\left(\Gamma^{12} \partial_{t}-\Gamma^{02} \partial_{1}+\Gamma^{01} \partial_{2}\right) \Psi=0
$$

Then rescaling as in the previous subsection, we see that the equation just acquires an overall factor of $c$. However, we should replace the original gamma matrices with the operators obeying the Heisenberg commutation relations. Then we immediately obtain

$$
\left(\Theta \partial_{t}+\epsilon_{i j} \chi_{i} \partial_{j}\right) \Psi=0
$$

which in operator notation takes the form

$$
\left(\Theta H+\epsilon_{i j} K_{i} P_{j}\right) \Psi=0
$$

Non-relativistic limits of the Dirac equation have been studied in the literature. In particular, two well-studied equations are the Pauli equation and the Lévy-Leblond equation [42]. However, the equation (3.12) is not equivalent to these 7 . In particular, notice that this is an infinite-component equation.

We will see in Sec. 4 that the same equation arises by considering a field which saturates the unitarity bound. This is analogous to the Schrödinger case, where fields

\footnotetext{
${ }^{7}$ As far as we are aware, an equation closely related to (3.12) was presented in [29. However, the discussion there assumes a non-zero mass $m$.
} 
saturating the unitarity bound obey the free Schrödinger equation, which in turn arises from the DLCQ of the Klein-Gordon equation. We regard equation (3.12) as the counterpart of the free Schrödinger equation in the present case, and we will discuss it further in Sec. 5 ,

\section{Properties of CFTs with exotic conformal Galilei symmetry}

\subsection{Action on operators}

Local operators $\mathcal{O}(t, \vec{x})$ depending on position in time and space are defined as

$$
\mathcal{O}(t, \vec{x})=\mathrm{e}^{-H t-P_{i} x_{i}} \mathcal{O}(0) \mathrm{e}^{H t+P_{i} x_{i}} .
$$

Such operators may be labeled by their scaling dimension and $\Theta$-quantum number

$$
[\mathcal{O}, D]=\Delta_{\mathcal{O}} \mathcal{O}, \quad[\mathcal{O}, \Theta]=i \theta_{\mathcal{O}} \mathcal{O}
$$

and in general form a representation of the exotic conformal Galilei algebra. Notice that $\left[\mathcal{O}, P_{i}\right]$ and $[\mathcal{O}, H]$ have scaling dimension $\Delta_{\mathcal{O}}+1,\left[\mathcal{O}, K_{i}\right]$ has unchanged scaling dimension $\Delta_{\mathcal{O}}$, while $\left[\mathcal{O}, F_{i}\right]$ and $[\mathcal{O}, C]$ have scaling dimensions $\Delta_{\mathcal{O}}-1$. Thus it is natural to define as primary operators those satisfying

$$
\left[\mathcal{O}, F_{i}\right]=[\mathcal{O}, C]=0
$$

From a primary operator it is possible to construct a tower of operators by acting with $H, P_{i}$, and $K_{i}$, forming an irreducible representation of the algebra.

When the operator $\mathcal{O}$ is inserted at a generic point, one finds the following action of the exotic conformal Galilei symmetry:

$$
\begin{aligned}
& {[\mathcal{O}, H]=\partial_{t} \mathcal{O}} \\
& {\left[\mathcal{O}, P_{i}\right]=\frac{\partial}{\partial x_{i}} \mathcal{O}} \\
& {[\mathcal{O}, D]=\left(-t \frac{\partial}{\partial t}-x_{i} \frac{\partial}{\partial x_{i}}-\Delta\right) \mathcal{O}} \\
& {\left[\mathcal{O}, K_{i}\right]=\left(-t \frac{\partial}{\partial x_{i}}+\chi_{i}\right) \mathcal{O}} \\
& {[\mathcal{O}, C]=\left(t^{2} \frac{\partial}{\partial t}+2 t x_{i} \frac{\partial}{\partial x_{i}}-2 x_{i} \chi_{i}+2 t \Delta\right) \mathcal{O}, \quad\left[\mathcal{O}, F_{i}\right]=\left(t^{2} \frac{\partial}{\partial x_{i}}-2 t \chi_{i}+2 i \theta x_{j} \epsilon_{i j}\right) \mathcal{O},} \\
& {[\mathcal{O}, J]=\left(-\epsilon_{i j} x_{i} \frac{\partial}{\partial x_{j}}-\frac{1}{2 i \theta} \chi_{i} \chi_{i}\right) \mathcal{O}}
\end{aligned}
$$

Note that the operator $\hat{K}_{i}=K_{i}+t P_{i}$ commutes with $H$ and $P_{i}$ once we identify $H$ with $\partial_{t}$ as above. Then we can introduce extra directions $v_{1,2}$ by defining

$$
\mathcal{O}(t, \vec{x}, \vec{v})=\mathrm{e}^{-\hat{K}_{i} v_{i}} \mathcal{O}(t, \vec{x}) \mathrm{e}^{\hat{K}_{i} v_{i}}
$$


Let us see how the generators act in this representation. Using the Zassenhaus formula, we have

$$
\mathcal{O}(t, \vec{x}, \vec{v})=\mathrm{e}^{i \theta v_{1} v_{2} / 2} \mathrm{e}^{-\hat{K}_{1} v_{1}} \mathrm{e}^{-\hat{K}_{2} v_{2}} \mathcal{O}(t, \vec{x}) \mathrm{e}^{\hat{K}_{2} v_{2}} \mathrm{e}^{\hat{K}_{1} v_{1}}
$$

Then, taking the derivative with respect to $v_{i}$, we get

$$
\left[\mathcal{O}(t, \vec{x}, \vec{v}), \hat{K}_{i}\right]=\left(\frac{\partial}{\partial v_{i}}-\frac{i \theta}{2} \epsilon_{i j} v_{j}\right) \mathcal{O}(t, \vec{x}, \vec{v}) .
$$

This shows that on local operators (4.5) the action of $\chi_{i}$ in (4.4) is given by

$$
\chi_{i}=\frac{\partial}{\partial v_{i}}-\frac{i \theta}{2} \epsilon_{i j} v_{j}
$$

\section{$4.2 \quad$ State-operator correspondence}

It is well-known that in relativistic conformal field theories there exists a one-to-one correspondence between operators and states. One way to establish this is to perform radial quantization. In [3] an analogous correspondence was worked out for nonrelativistic CFTs with Schrödinger symmetry. As we show below, this construction generalizes straightforwardly to higher $l$, because it is based on the common $S L(2 ; \mathbb{R})$ sub-algebras. For simplicity we will concentrate on the $l=1$ case.

We define a state $\left|\Psi_{\mathcal{O}}\right\rangle$ associated to the operator $\mathcal{O}$ via the formula

$$
\left|\Psi_{\mathcal{O}}\right\rangle=\mathrm{e}^{\frac{\pi i}{4}(C-H)} \mathcal{O}|0\rangle
$$

where $|0\rangle$ is the vacuum, defined as a state which satisfies

$$
G|0\rangle=0
$$

for all generators $G$. Here we think of $\mathcal{O}$ as a creation operator. If $\mathcal{O}$ is a primary, this formula reduces to

$$
\left|\Psi_{\mathcal{O}}\right\rangle=\mathrm{e}^{-\frac{\pi i}{4} H} \mathcal{O}|0\rangle
$$

which was used in [3]. However (4.9) holds more generally. Using the relation

$$
\tilde{D} \equiv \mathrm{e}^{\frac{\pi i}{4}(C-H)} D \mathrm{e}^{-\frac{\pi i}{4}(C-H)}=\frac{i}{2}(H+C)
$$

it is easy to check that $\left|\Psi_{\mathcal{O}}\right\rangle$ has definite scaling dimension, namely it obeys

$$
\tilde{D}\left|\Psi_{\mathcal{O}}\right\rangle=i \Delta_{\mathcal{O}}\left|\Psi_{\mathcal{O}}\right\rangle
$$


Since the angular momentum $J$ commutes with dilatations $D$, we can label highest weight states as $|\theta, \Delta, j\rangle$, where $j$ is the angular momentum quantum number. In the Schrödinger case such representations have been discussed in detail in [43] and are similarly characterized by $|m, \Delta, j\rangle$.

\subsection{Unitarity bound}

It is now easy to determine a lower bound on the scaling dimension of highest weight states imposed by unitarity. For the Schrödinger case this analysis has been done in [43, 44]. We do the analysis explicitly for the $l=1$ case, but the method we employ is applicable more generally. In particular, we use the transformation (4.12) to construct a non-unitary representation of the algebra, in terms of new generators

$$
\tilde{G}=\mathrm{e}^{\frac{\pi i}{4}(C-H)} G \mathrm{e}^{-\frac{\pi i}{4}(C-H)} .
$$

This is an automorphism of the algebra which manifestly preserves the commutation relations, but it changes their Hermiticity properties. Let us record the transformed generators

$$
\begin{aligned}
& \tilde{H}=\frac{1}{2}(H-C+2 i D), \quad \tilde{D}=\frac{i}{2}(H+C), \quad \tilde{C}=\frac{1}{2}(-H+C+2 i D), \\
& \tilde{P}_{i}=\frac{1}{2}\left(P_{i}-F_{i}+2 i K_{i}\right), \quad \tilde{K}_{i}=\frac{i}{2}\left(P_{i}+F_{i}\right), \quad \tilde{F}_{i}=\frac{1}{2}\left(-P_{i}+F_{i}+2 i K_{i}\right) .
\end{aligned}
$$

Notice that $\tilde{K}_{i}^{\dagger}=\tilde{K}_{i}, \tilde{F}_{i}^{\dagger}=\tilde{P}_{i}$ and $\tilde{\Theta}^{\dagger}=-\tilde{\Theta}$. Now we consider the state

$$
|\psi\rangle=\left(c \tilde{\Theta} \tilde{H}+\epsilon_{i j} \tilde{K}_{i} \tilde{P}_{j}\right)|\phi\rangle
$$

for an arbitrary real constant $c$. We also take $|\phi\rangle$ to be a primary, namely

$$
\tilde{F}_{i}|\phi\rangle=\tilde{C}|\phi\rangle=0
$$

A short computation than gives the norm

$$
\langle\psi \mid \psi\rangle=\theta^{2}\langle\phi \mid \phi\rangle 2\left(c^{2} \Delta-2 c+1\right) \geq 0
$$

We find the scaling dimension must satisfy

$$
\Delta \geq \frac{2 c-1}{c^{2}} .
$$

The right hand side is maximized at $c=1$, thus we get the bound

$$
\Delta \geq 1
$$


States $\left|\psi_{\text {free }}\right\rangle$ saturating this bound then obey the equation

$$
\left(\tilde{\Theta} \tilde{H}+\epsilon_{i j} \tilde{K}_{i} \tilde{P}_{j}\right)\left|\psi_{\text {free }}\right\rangle=0
$$

which is the analogue of a free field obeying the Schrödinger equation in the $l=1 / 2$ case. This corresponds to a field $\psi_{\text {free }}$ satisfying

$$
\left(\Theta H+\epsilon_{i j} K_{i} P_{j}\right) \psi_{\text {free }}=0 .
$$

Remarkably this is exactly the equation (3.12) obtained from the improved contraction of the Dirac equation. We will study the free field theory associated to this equation in Sec. 5 .

\subsection{Two-point functions}

In relativistic CFTs as well as non-relativistic CFTs, correlation functions are constrained by Ward identities. The form of correlation functions of non-relativistic CFTs with Schrödinger invariance was analyzed in [33]. For example, two-point functions are determined uniquely to be of the form

$$
\left\langle\mathcal{O}_{1}(t, \vec{x}), \mathcal{O}_{2}(0, \overrightarrow{0})\right\rangle=C \delta_{\Delta_{1}, \Delta_{2}} \delta_{M_{1}, M_{2}} \theta(t) t^{-\Delta_{1}} \exp \left(i \frac{M_{1}}{2} \frac{\vec{x}^{2}}{t}\right)
$$

where $C$ is a constant. To have a non-zero two-point function, the scaling dimensions and masses of the two local operators must be equal: $M_{1}=M_{2}$ and $\Delta_{1}=\Delta_{2}$.

Here we will repeat the analysis for the case at hand, namely the $l=1$ exotic conformal Galilei symmetry. We follow closely the exposition of [33]. We consider the two-point function of local operators (4.5) in the extended space-time

$$
F=\left\langle\mathcal{O}_{1}\left(t_{1}, \vec{x}_{1}, \vec{v}_{1}\right) \mathcal{O}_{2}\left(t_{2}, \vec{x}_{2}, \vec{v}_{2}\right)\right\rangle \text {. }
$$

We then consider in turn the constraints imposed by the symmetries, where the action of the operators on $F$ is given by the sum of the actions in (4.4), specified in the representation (2.32). It is convenient to define the variables

$$
\begin{aligned}
& t=t_{1}-t_{2}, \quad \vec{x}=\vec{x}_{1}-\vec{x}_{2}, \quad \vec{v}=\overrightarrow{v_{1}}-\overrightarrow{v_{2}}, \\
& t_{+}=t_{1}+t_{2}, \quad \vec{x}_{+}=\vec{x}_{1}+\vec{x}_{2}, \quad \vec{v}_{+}=\overrightarrow{v_{1}}+\overrightarrow{v_{2}} \text {. }
\end{aligned}
$$

First of all, time and space translations imply that the correlation functions must be functions of $t, \vec{x}$, but in principle also of both $\vec{v}, \vec{v}_{+}$. Dilatation invariance then implies

$$
\left(t \frac{\partial}{\partial t}+x_{i} \frac{\partial}{\partial x_{i}}+\Delta_{+}\right) F\left(t, \vec{x}, \vec{v}, \vec{v}_{+}\right)=0
$$


where $\Delta_{+}=\Delta_{1}+\Delta_{2}$. Defining $\vec{u}=\vec{x} / t$, we find that

$$
F\left(t, \vec{x}, \vec{v}, \vec{v}_{+}\right)=t^{-\Delta_{+}} G\left(\vec{u}, \vec{v}, \vec{v}_{+}\right) .
$$

Invariance under boosts gives

$$
\left(\frac{\partial}{\partial u_{i}}-2 \frac{\partial}{\partial v_{+i}}+\frac{i}{4} \epsilon_{i j}\left(v_{+j} \theta_{+}+v_{j} \theta_{-}\right)\right) G\left(\vec{u}, \vec{v}, \vec{v}_{+}\right)=0
$$

where $\theta_{ \pm}=\theta_{1} \pm \theta_{2}$. Now, let us consider the action of accelerations $F_{i}$. Changing variables, and hitting the equation with $\partial / \partial x_{+i}$, we find that $\theta_{+} G\left(\vec{u}, \vec{v}, \vec{v}_{+}\right)=0$, namely the "selection rule"

$$
\theta_{2}=-\theta_{1}
$$

This is analogous to $M_{1}=M_{2}$ in the Bargmann group [33]. Substituting this back in (4.28), we then find the following Ward identity

$$
\left(\frac{\partial}{\partial u_{i}}-2 \frac{\partial}{\partial v_{+i}}+i \frac{\theta_{1}}{2} \epsilon_{i j} v_{j}\right) G\left(\vec{u}, \vec{v}, \vec{v}_{+}\right)=0 .
$$

Plugging this into the equation coming from accelerations we determine a second Ward identity

$$
\left(\frac{\partial}{\partial v_{i}}-i \theta_{1} \epsilon_{i j}\left(u_{j}+\frac{1}{4} v_{+j}\right)\right) G\left(\vec{u}, \vec{v}, \vec{v}_{+}\right)=0,
$$

which can be immediately integrated to give

$$
G\left(\vec{u}, \vec{v}, \vec{v}_{+}\right)=g\left(\vec{u}, \vec{v}_{+}\right) \mathrm{e}^{i \theta_{1} \epsilon_{i j} v_{i}\left(u_{j}+\frac{1}{4} v_{+j}\right)} .
$$

Plugging this into (4.30) we get

$$
\left(\frac{\partial}{\partial u_{i}}-2 \frac{\partial}{\partial v_{+i}}\right) g\left(\vec{u}, \vec{v}_{+}\right)=0 .
$$

Therefore we have

$$
G\left(\vec{u}, \vec{v}, \vec{v}_{+}\right)=g\left(\vec{u}+\frac{\vec{v}_{+}}{2}\right) \mathrm{e}^{i \theta_{1} \epsilon_{i j} v_{i}\left(u_{j}+\frac{1}{4} v_{+j}\right)} .
$$

Next, requiring invariance of $F\left(t, \vec{x}, \vec{v}, \vec{v}_{+}\right)$under the conformal transformation $C$ and using (4.30) we obtain

$$
\left(-2 x_{i} \frac{\partial}{\partial v_{i}}+i \frac{\theta_{1}}{2} \epsilon_{i j} x_{i} v_{+j}+t\left(\Delta_{1}-\Delta_{2}\right)\right) G\left(\vec{u}, \vec{v}, \vec{v}_{+}\right)=0
$$


and contracting (4.31) with $x_{i}$ we find that the only new constraint it imposes is

$$
\Delta_{1}=\Delta_{2}
$$

It remains to check rotation invariance. The rotation generator acting on one operator is given in (2.30). When acting on two-point functions, we have

$$
J=J_{1}+J_{2}=-\epsilon_{i j}\left(x_{i} \frac{\partial}{\partial x_{j}}+\frac{1}{2} v_{i} \frac{\partial}{\partial v_{j}}+\frac{1}{2} v_{+i} \frac{\partial}{\partial v_{+j}}\right)+\frac{2 i}{\theta} \frac{\partial}{\partial v_{i}} \frac{\partial}{\partial v_{+i}}-i \frac{\theta}{8} v_{i} v_{+i},
$$

which is

$$
J=\mathrm{e}^{i \theta_{1} \epsilon_{i j} v_{i}\left(u_{j}+\frac{1}{4} v_{+j}\right)}\left(-\epsilon_{i j} u_{i}\left(\frac{\partial}{\partial u_{j}}-2 \frac{\partial}{\partial v_{+j}}\right)-\epsilon_{i j} v_{i} \frac{\partial}{\partial v_{j}}+\frac{2 i}{\theta} \frac{\partial}{\partial v_{i}} \frac{\partial}{\partial v_{+i}}\right) \mathrm{e}^{-i \theta_{1} \epsilon_{i j} v_{i}\left(u_{j}+\frac{1}{4} v_{+j}\right)} .
$$

Thus we find that (4.34) is invariant under this.

In conclusion, we have proved that the general two-point function is

$$
F=\delta_{\Delta_{1}, \Delta_{2}} \delta_{\theta_{1},-\theta_{2}} t^{-2 \Delta_{1}} g\left(\vec{u}+\frac{\vec{v}_{+}}{2}\right) \exp \left(i \theta_{1} \vec{v} \times\left(\vec{u}+\frac{1}{4} \vec{v}_{+}\right)\right)
$$

where we used a shorthand notation $\vec{a} \times \vec{b} \equiv \epsilon_{i j} a_{i} b_{j}$. Notice the $t^{-2 \Delta_{1}}$ dependence, as opposed to $t^{-\Delta_{1}}$ in the Schrödinger case (4.23). This is of course correct, since the two-point function has scaling dimension $2 \Delta_{1}$, and $t$ has dimension one here, while it has dimension two in the Schrödinger case.

\section{Field theories with exotic conformal Galilei sym- metry}

\subsection{Free field action}

In Sec. 4 we showed that a field saturating the unitarity bound $\Delta=1$ satisfies equation (3.12), arising also from the non-relativistic contraction of the massless Dirac equation. This is the equation of motion of the free field action

$$
S=\int \mathrm{d} t \mathrm{~d}^{2} \vec{x}\left(\bar{\psi},\left(\partial_{t}+\frac{1}{i \theta} \epsilon_{i j} \chi_{i} \partial_{j}\right) \psi\right)
$$

where $(\bar{\phi}, \psi)$ is an inner product on the space on which $\chi_{i}$ act, so that $\chi_{i}$ is antiHermitean. When we use $v_{1,2}$ to represent $\chi_{1,2}$, it is given by the integration over $v_{1,2}$, i.e.

$$
(\bar{\phi}, \psi)=\int \mathrm{d}^{2} \vec{v} \bar{\phi} \psi
$$


We can check that this action is indeed invariant under the exotic conformal Galilei symmetry, (4.4) when $\Delta=1$. Invariance under $H, P_{i}, D$ is immediate. Invariance under $C$ then implies invariance under $K_{i}$ and $F_{i}$, so let us check that. The change of the action is, using integration by parts,

$$
\begin{aligned}
\int \mathrm{d} t \mathrm{~d}^{2} \vec{x} & {\left[\left(\overline{C \psi},\left(\partial_{t}+\frac{1}{i \theta} \epsilon_{i j} \chi_{i} \partial_{j}\right) \psi\right)+\left(\bar{\psi},\left(\partial_{t}+\frac{1}{i \theta} \epsilon_{i j} \chi_{i} \partial_{j}\right) C \psi\right)\right] } \\
& =\int \mathrm{d} t \mathrm{~d}^{2} x\left[2 t\left(\bar{\psi},\left(\partial_{t}+\frac{1}{i \theta} \epsilon_{i j} \chi_{i} \partial_{j}\right) \psi\right)-\left(\bar{\psi},\left[C, \partial_{t}+\frac{1}{i \theta} \epsilon_{i j} \chi_{i} \partial_{j}\right] \psi\right)\right] .
\end{aligned}
$$

Now, an explicit calculation shows

$$
\left[C, \partial_{t}+\frac{1}{i \theta} \epsilon_{i j} \chi_{i} \partial_{j}\right]=2 t\left(\partial_{t}+\frac{1}{i \theta} \epsilon_{i j} \chi_{i} \partial_{j}\right)
$$

therefore we conclude this action is invariant under the exotic conformal symmetry.

When the operators $\chi_{1,2}$ and $\Theta$ are realized using the coordinates $v_{1,2}$ and $\xi$, the action above becomes

$$
\begin{aligned}
S & =-\int \mathrm{d} t \mathrm{~d} \xi \mathrm{d}^{2} \vec{x} \mathrm{~d}^{2} \vec{v}\left(\partial_{\xi} \bar{\psi} \partial_{t}+\epsilon_{i j} \chi_{i} \bar{\psi} \partial_{j} \psi\right) \\
& =-\int \mathrm{d}^{6} x \sqrt{-g} g^{I J} \partial_{I} \bar{\psi} \partial_{J} \psi
\end{aligned}
$$

where $I$ runs from 1 to 6 , corresponding to the coordinates $t, \xi, x_{1}, x_{2}, v_{1}, v_{2}$, and the metric is given by

$$
\mathrm{d} s^{2}=\mathrm{d} t\left(\mathrm{~d} \xi-\frac{1}{2} \epsilon_{i j} v_{i} \mathrm{~d} v_{j}\right)-\epsilon_{i j} \mathrm{~d} x_{i} \mathrm{~d} v_{j}
$$

This is in fact a metric on flat $\mathbb{R}^{3,3}$. Defining

$$
y_{i}=x_{i}+\frac{1}{2} t v_{i}
$$

the metric becomes

$$
\mathrm{d} s^{2}=\mathrm{d} t \mathrm{~d} \xi-\epsilon_{i j} \mathrm{~d} y_{i} \mathrm{~d} v_{j} .
$$

One finds that the vector fields (2.37) acts on this flat metric as a conformal transformation. Then the invariance of the action (5.5) with a suitable weight on $\psi$ is automatic, because a free relativistic scalar field on a flat space is relativistically conformally invariant.

This is clearly parallel to the fact that the invariance of the action of a free nonrelativistic massive particle

$$
S=\int \mathrm{d} t \mathrm{~d}^{d} x \bar{\psi}\left(i \partial_{t}+\frac{1}{2 m} \partial_{i} \partial_{i}\right) \psi
$$


under the Schrödinger symmetry. Indeed, by introducing the direction $\xi$ conjugate to $m$, the action can be written as

$$
S=-\int \mathrm{d} t \mathrm{~d} \xi \mathrm{d}^{d} x\left(2 \partial_{\xi} \bar{\psi} \partial_{t} \psi+\partial_{i} \bar{\psi} \partial_{i} \psi\right)
$$

and this describes a free relativistic particle on a flat space $\mathbb{R}^{d+1,1}$ with the metric (2.9) .

\subsection{Chern-Simons-matter action}

The free field action can be coupled to a $U(1)$ gauge field, together with a Chern-Simons term, without spoiling the Galilei conformal invariance, at least classically. The total action is

$$
S=\int \mathrm{d} t \mathrm{~d}^{2} \vec{x}\left[-\frac{\lambda}{2} \epsilon_{a b c} A_{a} \partial_{b} A_{c}+\left(\bar{\psi},\left(D_{t}+\frac{1}{i \theta} \epsilon_{i j} \chi_{i} D_{j}\right) \psi\right)\right],
$$

where $A_{a}$ is the gauge field and $\lambda$ is the Chern-Simons level. We use the convention that the indices $a, b, c=0,1,2$ stand for the directions $t, x_{1}, x_{2}$ combined. $D_{a}$ is the covariant derivative

$$
D_{a}=\partial_{a}+i A_{a}
$$

Note that $A_{a}$ only depends on $t, x_{1,2}$, and we do not have components $A_{v_{i}}$ or $A_{\xi}$. The conformal Galilei group acts on $A_{a}$ by the Lie derivative $£$ via the vector fields (2.14). Being topological, the Chern-Simons term is automatically invariant under the conformal Galilei group. We let the exotic conformal Galilei group act on $\psi$ as before, i.e. without changing $\partial_{a}$ to $D_{a}$ in (4.4). Invariance of the action under $H, P_{i}$ and $D_{i}$ is again trivial. The change under $C$ of the Lagrangian is now

$$
\begin{aligned}
\int \mathrm{d} t \mathrm{~d}^{2} \vec{x} & 2 t\left(\bar{\psi},\left(D_{t}+\frac{1}{i \theta} \epsilon_{i j} \chi_{i} D_{j}\right) \psi\right) \\
& \left.-\left(\bar{\psi},\left[C, D_{t}+\frac{1}{i \theta} \epsilon_{i j} \chi_{i} D_{j}\right] \psi\right)-\left(\bar{\psi},\left[\left(£_{C} A\right)_{t}+\frac{1}{i \theta} \epsilon_{i j} \chi_{i}\left(£_{C} A\right)_{j}\right] \psi\right)\right]
\end{aligned}
$$

which vanishes using the fact that

$$
\left[C, D_{a}\right]+\left(£_{C} A\right)_{a}=2 t D_{a}
$$

Therefore the action is invariant under $C$ and invariance under $K_{i}$ and $F_{i}$ follows automatically.

The equations of motion for the gauge field are then

$$
F_{12}=\frac{i}{\lambda}(\bar{\psi}, \psi), \quad F_{0 i}=\frac{i}{\lambda \theta}\left(\bar{\psi}, \chi_{i} \psi\right)
$$


Let us stress again that the gauge potential $A_{a}$ and the field strength $F_{a b}$ only depend on $t, x_{1,2}$. When we represent $\chi_{i}$ using $v_{i}$, the pairing in the right hand side of (5.15) involves the integration along the $v_{i}$ directions, e.g.

$$
F_{12}=\frac{i}{\lambda} \int \mathrm{d}^{2} \vec{v} \bar{\psi} \psi
$$

It would be nice to study the coupled CS-matter system in more detail, but we leave this for future work, and return to discuss the free model.

\subsection{Free field wave function}

We will now construct simple solutions to the free field equation (3.12) in the representation (2.32), analogous to the plane-wave solutions of the Schrödinger equation 8 . In particular, we will discuss the transformation properties under the exotic conformal symmetry group, showing that the wave functions are projective representations of this group. These solutions will be also useful later in the "holographic" calculation in Sec. 6.

The quickest way to arrive at the solution is to employ a change of coordinates which manifestly transforms the operator $\Theta H+\vec{K} \times \vec{P}$ to the Laplacian on $\mathbb{R}^{3,3}$ as we used in sub-section 5.1. Using (5.7) the wave equation becomes

$$
\left[\frac{\partial}{\partial \xi} \frac{\partial}{\partial t}+\epsilon_{i j} \frac{\partial}{\partial v_{i}} \frac{\partial}{\partial y_{j}}\right] \psi=0 .
$$

The solutions are spanned by plane waves

$$
\psi_{E, \vec{p}, \vec{k}}=\exp i\left(\theta \xi+E t-\vec{p} \cdot \vec{y}-\frac{1}{2} \vec{k} \cdot \vec{v}\right), \quad E=\frac{1}{2 \theta}(\vec{p} \times \vec{k})
$$

which in the original coordinates read

$$
\psi_{E, \vec{p}, \vec{k}}=\exp i\left(\theta \xi+E t-\vec{p} \cdot \vec{x}-\frac{1}{2}(\vec{k}+t \vec{p}) \cdot \vec{v}\right) .
$$

This shows that the parameter $\theta$ behaves as "mass" for a Schrödinger equation in $\mathbb{R}^{2,2}$.

Let us now address invariance of this solution under the conformal Galilei symmetries. The finite boost $K(\vec{b})$ transformation (2.39) acts on the wavefunction as

$$
U_{K(\vec{b})} \psi(X)=\psi(K(-\vec{b}) X)
$$

\footnotetext{
${ }^{8}$ Other representations of the Heisenberg generators $\chi_{i}$ are possible, but we will not consider them here.
} 
where $X$ stands for a point in the extended space time, $X=(t, \xi, \vec{x}, \vec{y})$. It acts on the plane waves as

$$
U_{K(\vec{b})} \psi_{E, \vec{p}, \vec{k}}=\mathrm{e}^{\frac{i}{2} \vec{k} \cdot \vec{b}} \psi_{E-\frac{1}{2} \vec{p} \cdot \vec{b}, \vec{p}, k_{i}+\theta \epsilon_{i j} b_{j}}
$$

i.e. one has

$$
p_{i} \rightarrow p_{i}, \quad k_{i} \rightarrow k_{i}+\theta \epsilon_{i j} b_{j}, \quad E \rightarrow E-\frac{1}{2} p_{i} b_{i}
$$

In particular, we see that while $\vec{p}$ is clearly the momentum associated to the wave function, $\vec{k}$ may be interpreted as the "boost". Indeed the transformations of $\vec{k}$ under the full conformal Galilei group are compatible with this interpretation. It is easy now to check that

$$
U_{K(\vec{b})} U_{K\left(\overrightarrow{b^{\prime}}\right)}=\mathrm{e}^{i \theta \vec{b} \times \vec{b}^{\prime}} U_{K\left(\vec{b}^{\prime}\right)} U_{K(\vec{b})}
$$

with the required phase term coming from the central charge $\left[K_{i}, K_{j}\right]=\epsilon_{i j} i \theta$. Next consider the accelerations. One can take (2.40) as the finite transformation, which we denote by $F(\vec{a})$. We then let it act on the wavefunction as

$$
U_{F(\vec{a})} \psi(X)=\psi(F(-\vec{a}) X)
$$

It acts on the plane wave as follows:

$$
U_{F(\vec{a})} \psi_{E, \vec{p}, \vec{k}}=\psi_{E-\vec{k} \cdot \vec{a}, p_{i}-2 \theta \epsilon_{i j} a_{j}, k_{i}},
$$

i.e. one has

$$
p_{i} \rightarrow p_{i}-2 \theta \epsilon_{i j} a_{j}, \quad k_{i} \rightarrow k_{i}, \quad E \rightarrow E-k_{i} a_{i}
$$

Recalling

$$
U_{P(\vec{c})} \psi_{E, \vec{p}, \vec{k}}=\mathrm{e}^{-i \vec{c} \cdot \vec{p}} \psi_{E, \vec{p}, \vec{k}},
$$

one can easily see that

$$
U_{P(\vec{c})} U_{F(\vec{a})}=\mathrm{e}^{-2 i \theta \vec{c} \times \vec{a}} U_{F(\vec{a})} U_{P(\vec{c})},
$$

thus realizing $\left[P_{i}, F_{j}\right]=-2 \epsilon_{i j} i \theta$. Let us finally address conformal transformations generated by $C$. The infinitesimal version is given in (2.37), and the integrated version is

$$
\begin{aligned}
t & \rightarrow \frac{t}{1-a t}, & x_{i} & \rightarrow \frac{x_{i}}{(1-a t)^{2}}, \\
v_{i} & \rightarrow v_{i}-\frac{2 x_{i}}{t(1-a t)}, & \xi & \rightarrow \xi+\frac{2}{t(1-a t)} \epsilon_{i j} x_{i} v_{j} .
\end{aligned}
$$


Let us denote this action by $C(a)$. This affects the metric (5.6) as

$$
\mathrm{d} s^{2} \rightarrow \frac{\mathrm{d} s^{2}}{(1-a t)^{2}} .
$$

Then the action on the wavefunction is

$$
U_{C(a)} \psi(X)=(1+a t)^{2} \psi(C(-a) X) .
$$

This does not map plane waves to plane waves, but it correctly maps solutions to solutions.

\subsection{Two-point functions}

It is straightforward calculate the two-point functions of the free theory in the $v_{i}$ representation. In the six-dimensional flat space, the two-point function of the relativistic massless free scalar field $\psi$ is given by

$$
\left\langle\psi\left(x_{(1)}^{I}\right) \psi\left(x_{(2)}^{I}\right)\right\rangle \propto\left|x_{(1)}^{I}-x_{(2)}^{I}\right|^{-4} .
$$

Using the coordinate change (5.7) from the $v_{i}$ representation to the flat $\mathbb{R}^{3,3}$, one finds

$$
\left\langle\psi\left(x_{(1)}^{I}\right) \psi\left(x_{(2)}^{I}\right)\right\rangle \propto\left(t \xi-\epsilon_{i j}\left(x_{i}+\frac{t v_{+j}}{4}\right) v_{j}\right)^{-2} .
$$

In order to compare this to the results in Sec. 4.4, one needs to perform the Fourier transformation along $\xi$. Then the two-point function is

$$
\int \mathrm{d} \xi e^{i \theta \xi}\left(t \xi-\epsilon_{i j}\left(x_{i}+\frac{t v_{+j}}{4}\right) v_{j}\right)^{-2}=t^{-2} \theta \exp \left(i \theta \epsilon_{i j}\left(u_{i}+\frac{v_{+j}}{4}\right) v_{j}\right) \int \mathrm{d} \xi e^{i \xi} \xi^{-2} .
$$

The last term is just an numerical coefficient. Indeed, this is exactly of the form (4.39), where $g\left(\vec{u}+\vec{v}_{+} / 2\right) \equiv 1$ and $\Delta=1$.

\section{Geometric realization}

\subsection{Invariant metric}

In the previous section we saw that one way to realize the free field with the exotic conformal Galilei invariance is to use a free "relativistic" scalar field on flat $\mathbb{R}^{3,3}$. The exotic conformal Galilei invariance acts on $\mathbb{R}^{3,3}$ as a relativistic conformal transformation. Then it is easy to add another direction to realize the whole symmetry as the isometry. Indeed, by taking the seven-dimensional metric

$$
g=\frac{1}{z^{2}}\left[\mathrm{~d} z^{2}-\mathrm{d} t\left(\mathrm{~d} \xi-\frac{1}{2} \epsilon_{i j} v_{i} \mathrm{~d} v_{j}\right)+\epsilon_{i j} \mathrm{~d} x_{i} \mathrm{~d} v_{j}\right]
$$


which is just a rewriting of " $\mathrm{AdS}_{7}$ " with $(3,4)$ signature, we can realize the exotic conformal Galilei algebra using isometries. The only generators modified by terms in $z$ are the dilatations and the conformal transformations. In particular, we have

$D=-x_{i} \frac{\partial}{\partial x_{i}}-t \partial_{t}-\frac{1}{2} z \partial_{z}, \quad C=2 t x_{i} \frac{\partial}{\partial x_{i}}-2 x_{i} \frac{\partial}{\partial v_{i}}+t^{2} \partial_{t}+\left(z^{2}+\epsilon_{i j} x_{i} v_{j}\right) \partial_{\xi}+t z \partial_{z}$

By analyzing general symmetric two-tensors invariant under the transformations (2.35) - (2.41), we find a three-parameter family of metrics, invariant under this set of isometries, namely:

$$
g=\frac{a}{z^{2}}\left[\mathrm{~d} z^{2}-\mathrm{d} t\left(\mathrm{~d} \xi-\frac{1}{2} \epsilon_{i j} v_{i} \mathrm{~d} v_{j}\right)+\epsilon_{i j} \mathrm{~d} x_{i} \mathrm{~d} v_{j}\right]+b \frac{\mathrm{d} t^{2}}{z^{4}}+\frac{c}{z^{4}}\left(\mathrm{~d} x_{i}+v_{i} \mathrm{~d} t\right)^{2} .
$$

Unfortunately, it turns out that the signature of this metric is always $(3,4)$. For this reason, we can not interpret this as bulk metric of a consistent gravity theory in seven dimensions.

Let us write down the Laplacian acting on scalars, constructed from the metric (6.3):

$$
\begin{aligned}
\square=\frac{z^{7}}{a} \frac{\partial}{\partial z}\left(\frac{1}{z^{5}} \frac{\partial}{\partial z}\right)-\frac{4 z^{2}}{a} & \left(\frac{\partial}{\partial t} \frac{\partial}{\partial \xi}+\epsilon_{i j} \chi_{i} \frac{\partial}{\partial x_{j}}\right)-\frac{4 b}{a^{2}} \frac{\partial^{2}}{\partial \xi^{2}} \\
& -\frac{4 c}{a^{2}}\left(\chi_{i} \chi_{i}-2 \epsilon_{i j} v_{i} \frac{\partial}{\partial v_{j}} \frac{\partial}{\partial \xi}\right)
\end{aligned}
$$

Notice that the second and third terms in the first line are the quadratic Casimir operators $H \Theta+\vec{K} \times \vec{P}$ and $\Theta^{2}$ of the exotic Galilei group (without conformal extension).

\section{2 "Holographic" two-point function}

Although the metric (6.3) has the wrong signature, recall that setting $b=c=0$, this is an analytic continuation of $\mathrm{AdS}_{7}$. Furthermore, when $c=0$, this metric is an analytic continuation of the metric which has the Schrödinger group as the isometry, constructed in [1, 2]. It is therefore natural to wonder whether applying the AdS/CFT rules to this metric might still give sensible results, in spite of the problematic signature. In the following, we then ignore this problem, and perform a holographic-type calculation of the two-point function. Holographic two- and three- point functions in Schrödinger backgrounds have been recently computed in [45, 46]. However, our treatment is more elementary and follows essentially the computation of two-point functions in [2].

We then imagine a massive scalar field propagating in the fixed background metric (6.3), and solve for its wave equation with fixed boundary conditions, following 
the original prescription [47, 48]. As we will see, as far as this naive computation is concerned, the wrong signature of the boundary metric does not play an important role.

For simplicity, we consider the metric (6.3) after setting $9=0$. We can set $a=1$ by an overall rescaling of the metric. The parameter $b$ may also be reabsorbed, but we leave it explicit. Using the ansatz

$$
\phi=\psi_{\text {free }}(t, \vec{x}, \xi, \vec{v}) \varphi(z)
$$

into the Klein-Gordon equation, we get

$$
-z^{7} \frac{\partial}{\partial z}\left(\frac{1}{z^{5}} \frac{\partial}{\partial z}\right) \varphi(z)+\kappa^{2} z^{2} \varphi(z)+\left(m^{2}-4 b \theta^{2}\right) \varphi(z)=0
$$

where we defined

$$
\kappa^{2} \equiv-4\left(E \theta+\frac{1}{2} \vec{k} \times \vec{p}\right)
$$

In particular, we require that $\kappa^{2}>0$. Defining

$$
\nu=\sqrt{9+m^{2}-4 b \theta^{2}}, \quad \hat{\Delta}=3+\nu
$$

the solution to (6.6), normalizable near the boundary $z \rightarrow 0$ is given by the Bessel function

$$
\varphi(z)=C z^{3} K_{\nu}(\kappa z)
$$

where $C$ is a constant. In particular, $\varphi \sim z^{\hat{\Delta}}$ for $z \rightarrow 0$. The reason why we decorate $\Delta$ with a hat will become clear momentarily. Notice that this solution is formally the same solution for the Klein-Gordon equation in a $\mathrm{Sch}_{4}$ background [2].

From the asymptotic expansion of (6.9) one extracts the "flux factor" at the boundary, which is essentially the Fourier transform of the two-point function in momentum space. For us "momentum space", means the space of $p=(E, \vec{p}, \theta, \vec{k})$. Thus the two-point function is

$$
\left\langle\mathcal{O}_{1}\left(t_{1}, \vec{x}_{1}, \xi_{1}, \vec{v}_{1}\right) \mathcal{O}_{2}\left(t_{2}, \vec{x}_{2}, \xi_{2}, \vec{v}_{2}\right)\right\rangle=\int \prod_{i=1}^{2} \mathrm{~d} \theta_{i} \mathrm{~d} E_{i} \mathrm{~d}^{2} \vec{p}_{i} \mathrm{~d}^{2} \vec{k}_{i}\left\langle\mathcal{O}_{1}\left(p_{1}\right) \mathcal{O}_{2}\left(p_{2}\right)\right\rangle \psi_{\text {free }_{1}} \psi_{\text {free }_{2}}
$$

\footnotetext{
${ }^{9}$ Solutions in the metric with $c \neq 0$ can also be studied, but they can not be expanded into the free wave functions as we do below.
} 
where $\left\langle\mathcal{O}_{1}\left(p_{1}\right) \mathcal{O}_{2}\left(p_{2}\right)\right\rangle$ is the momentum-space two-point function, and $\psi_{\text {free }}$ are the solutions (5.19). Making the change of variables $\vec{x} \rightarrow \vec{y}=\vec{x}+t \vec{v} / 2$ the free wave functions become simply the plane waves (5.18). It is then clear that solutions to the wave equation can be expanded into these plane waves as

$$
\phi(t, \vec{y}, \xi, \vec{v})=\int \mathrm{d} E \mathrm{~d} \theta \mathrm{d}^{2} \vec{p} \mathrm{~d}^{2} \vec{k} \hat{\phi}_{p} \psi_{\text {free }}
$$

and we have

$$
\left\langle\mathcal{O}_{1}\left(p_{1}\right) \mathcal{O}_{2}\left(p_{2}\right)\right\rangle=\delta\left(\theta_{1}+\theta_{2}\right) \delta\left(E_{1}+E_{2}\right) \delta^{2}\left(\vec{p}_{1}+\vec{p}_{2}\right) \delta^{2}\left(\vec{k}_{1}+\vec{k}_{2}\right)\langle\ldots\rangle
$$

where $\langle\ldots\rangle$ is extracted from the flux factor. Changing coordinates as in (4.25) we can carry out the integral over the delta-functions, reducing it to

$$
\int \mathrm{d} \theta_{1} \mathrm{~d} E_{1} \mathrm{~d} \vec{p}_{1} \mathrm{~d} \vec{k}_{1}\langle\ldots\rangle \mathrm{e}^{i\left[\theta_{1} \xi+E_{1} t-\vec{p}_{1} \cdot \vec{y}-\frac{1}{2} \vec{k}_{1} \cdot \vec{v}\right]}
$$

where notice that

$$
\vec{y}=\vec{x}+\frac{1}{4}\left(t \vec{v}_{+}+t_{+} \vec{v}\right) .
$$

We will now evaluate (6.13), where $\langle\ldots\rangle \propto \kappa^{2 \nu}$, without being concerned with the overall numerical coefficient. After the following change of variables

$$
z=t\left(E_{1}+\frac{1}{2 \theta_{1}} \vec{k}_{1} \times \vec{p}_{1}\right) \quad \vec{p}_{1}=\vec{p} \theta t^{-1 / 2} \quad \vec{k}_{1}=\vec{k} \theta t^{-1 / 2}
$$

we have

$$
\Gamma(\nu+1) t^{-\hat{\Delta}} \int \mathrm{d} \theta_{1} \theta_{1}^{\hat{\Delta}+1} \mathrm{e}^{i \theta_{1} \xi} \int \mathrm{d} \vec{p} \mathrm{~d} \vec{k} \exp \left[-i \theta_{1}\left(\frac{1}{2} \vec{k} \times \vec{p}+\frac{\vec{p} \cdot \vec{y}}{t^{1 / 2}}+\frac{\vec{k} \cdot \vec{v}}{2 t^{1 / 2}}\right)\right] .
$$

The latter integral is easily evaluated by analytically continuing $i \theta_{1} \rightarrow \theta_{1}$, making it just a Gaussian integral. In conclusion, we obtain

$\left\langle\mathcal{O}_{1}\left(t_{1}, \vec{x}_{1}, \xi_{1}, \vec{v}_{1}\right) \mathcal{O}_{2}\left(t_{2}, \vec{x}_{2}, \xi_{2}, \vec{v}_{2}\right)\right\rangle \propto t^{-\hat{\Delta}} \int \mathrm{d} \theta_{1} \theta_{1}^{\hat{\Delta}-1} \mathrm{e}^{i \theta_{1} \xi} \exp \left[i \theta_{1} \vec{v} \times\left(\frac{\vec{x}}{t}+\frac{1}{4} \vec{v}_{+}\right)\right]$

where notice that the dependence on $t_{1}+t_{2}$ through $\vec{y}$ dropped out of the final expression, as expected from invariance under time translations. We see that we get agreement with the general expression (4.39), provided we identify

$$
\hat{\Delta}=2 \Delta_{1}
$$


Indeed, notice that lower bound on $\hat{\Delta}$ is $\hat{\Delta}_{\text {min }}=2$. This corresponds to the unitarity bound for the free Schrödinger theory in $d=4$ dimensions, or equivalently for a relativistic CFT in $D=5+1$ dimensions. As an exotic Galilean conformal field theory in $d=2$ dimension, it correctly corresponds to the unitarity bound $\Delta_{\min }=1$.

The factor of $\theta_{1}^{2 \Delta-1}$ is analogous to the factor of $M_{1}^{\Delta-1}$ appearing in the holographic two-point function for Schrödinger theories [45].

\section{Conclusions}

In this paper we have discussed non-relativistic conformal systems with symmetries different from the Schrödinger type, and their geometric realizations. We started by analyzing a family of conformal Galilei algebras which are natural generalizations of the Schrödinger algebra. In particular, we have pointed out the existence of central extensions and infinite dimensional extensions. Our main interest was the Galilean algebra extended by constant accelerations and conformal transformations. This admits a central extension in $d=2$ spatial dimensions. Following analogous discussions of the Schrödinger algebra, we have studied generic properties of conformal field theories, with underlying conformal Galilei algebra. For example, we have defined primaries and discussed the general form of two-point functions. Higher $n$-point functions may be analyzed in a similar way. As the Schrödinger conformal field theories are relevant for studying cold atoms, our results should be useful for studying other, perhaps exotic, Galilean conformal field theories describing real physical systems [17].

One crucial ingredient in our approach is the central extension of the algebra. Such extension renders the boosts along the two directions in the plane non-commutative. Following [23], one way to understand the origin of this non-commutativity is to derive the algebra from a non-relativistic contraction of the relativistic conformal algebra, in a representation with non-zero spin. Although the contraction applied to an AdS metric is degenerate, we noticed that applying the modified contraction above to the (massless) Dirac equation leads a consistent non-trivial equation, also obeyed by fields whose scaling dimension saturates the unitarity bound. We have discussed that this equation is to the exotic conformal Galilei group what the free Schrödinger equation is to the Schrödinger group. Moreover, we have shown that one can couple it to a gauge field, together with a Chern-Simons term. This suggests that the contraction we have discussed may be applied to more complicated models, perhaps supersymmetric, like the model of [49]. It would be nice to see whether new interesting non-relativistic 
models may be obtained in this way.

Following the strategy that successfully lead to the Schrödinger geometries in [1, 2, we have presented a three-parameter metric that realizes the exotic Galilean symmetry group as its group of isometries. However, it turns out that this metric has not Lorentzian signature, but rather it has $(3,4)$ signature. Despite this, the geometric structure may be interpreted formally in AdS/CFT language, and leads to a modification of the " $\mathrm{AdS}_{7}$ " metric, where the boundary is $\mathbb{R}^{3,3}$. The three extra directions $(\xi, \vec{v})$ are associated to the central extension $\Theta$, and to velocities, respectively. The symmetries act on this space, in a slightly unusual way. For example, dilatations act as $D:(t, \vec{x}, \xi, \vec{v}) \rightarrow(\lambda t, \lambda \vec{x}, \xi, \vec{v})$.

Although this is a natural geometric (Bargmann-like) structure generalizing the Schrödinger case, the resulting metric is not suitable for AdS/CFT applications. Nevertheless, we have pointed out that a blind application of the AdS/CFT prescriptions gives a consistent "holographic" two-point function. We suspect that this feature will persist for three- and higher point functions. It would be interesting to see whether the geometric realization that we have presented, may be still useful at least as a tool for performing other holographic-type calculations.

The problem of finding a gravity dual of non-Schrödinger Galilean conformal field theories remains largely open. From our analysis, it emerged that the inclusion of fermionic variables in taking the non-relativistic limit plays an important role. Thus, one can try to obtain a bulk dual by applying the non-relativistic contraction to the matter-coupled supergravity equations, presumably including fermionic fields. A different approach to a geometric realization of the conformal Galilei algebra (without central extension) has been proposed in [30]. In this reference it is argued that the gravity side should be described by a version of Newton-Cartan theory, where the dynamical variables are non-metric connections. However, the relation of this approach to the AdS/CFT correspondence remains to be clarified. It should be interesting to investigate the relationship of our results to those of [30].

\section{Acknowledgments}

YT is supported in part by the NSF grant PHY-0503584, and in part by the Marvin L. Goldberger membership in the Institute for Advanced Study. 


\section{A Mechanics with exotic conformal Galilei symme- try}

The Lagrangian

$$
L=\frac{1}{2} m \dot{x}_{i}^{2}-\theta \epsilon_{i j} \dot{x}_{i} \ddot{x}_{j}
$$

is known to be invariant under the Galilei group with two central extensions $M$ and $\Theta$ [40]. If $\theta=0$, it is quasi-invariant under the Schrödinger group, meaning that it is invariant up to a total derivative. It is quasi-invariant under the exotic conformal Galilei group only if $m=0$, when the Lagrangian is

$$
L=-\theta \epsilon_{i j} \dot{x}_{i} \ddot{x}_{j}
$$

whose equation of motion is

$$
\dddot{x}_{i}=0
$$

We see that the solutions are constantly accelerating trajectories, which is analogous to the constant velocity trajectories solutions to $L=\frac{m}{2} \dot{x}_{i}^{2}$. Because of the high number of derivatives it is not easy to quantize it directly. Let us instead consider the Lagrangian

$$
L=-\theta\left(\epsilon_{i j} \dot{x}_{i} \dot{v}_{j}+\frac{1}{2} \epsilon_{i j} v_{i} \dot{v}_{j}\right) .
$$

The equations of motion of this are

$$
\dot{v}_{i}=-\ddot{x}_{i}, \quad \ddot{v}_{i}=0
$$

thus we see that this Lagrangian is equivalent to (A.2), and $v_{i}$ are essentially (minus) the velocities.

One way to rewrite this Lagrangian is to use the metric (5.6). Let $x^{I}$ denote $\left(t, \xi, x_{1}, x_{2}, v_{1}, v_{2}\right)$ collectively, and consider a free, massless "relativistic" particle moving in $\mathbb{R}^{3,3}$, with the standard action

$$
\int \mathrm{d} \tau e^{-1}(\tau) g_{I J}(x) \frac{\mathrm{d} x^{I}}{\mathrm{~d} \tau} \frac{\mathrm{d} x^{J}}{\mathrm{~d} \tau}
$$

where $\tau$ is the parameter of the worldline $x^{I}(\tau)$, and $e(\tau)$ is the einbein. This is invariant under the relativistic conformal group acting on $x^{I}$, which includes the exotic 
conformal Galilei group, because the conformal factor can be reabsorbed by the einbein. Now let us gauge-fix the worldline by choosing $\tau=t$, which gives the action

$$
-\int \mathrm{d} t e^{-1}\left[-\dot{\xi}+\frac{1}{2} \epsilon_{i j} v_{i} \dot{v}_{j}+\epsilon_{i j} \dot{x}_{i} \dot{v}_{j}\right] .
$$

Taking the variation with respect to $\xi$ one finds the einbein $e^{-1}$ is constant which we denote by $\theta$, resulting in

$$
-\theta \int \mathrm{d} t\left[-\dot{\xi}+\frac{1}{2} \epsilon_{i j} v_{i} \dot{v}_{j}+\epsilon_{i j} \dot{x}_{i} \dot{v}_{j}\right] .
$$

which is equivalent to (A.4) up to an apparently decoupled degree of freedom $\xi$; more precisely, the equation of motion associated to $e$ in (A.7) determines the time evolution of $\xi$ in terms of that of $x_{i}$ and $v_{i}$. One can check that the Lagrangian (A.4) without $\xi$ is invariant under the conformal Galilei transformation up to a total derivative, which is precisely absorbed by a shift of $\xi$ given in (2.42).

For comparison, it is instructive to carry out the same analysis of the conformal symmetry to the case of free non-relativistic particle with mass $m$. Let us consider again the Lagrangian of a massless relativistic particle (A.6), this time with the Bargmann metric (2.9) as $g_{I J}$. Gauge-fixing by $\tau=t$, one obtains the Lagrangian

$$
m \int \mathrm{d} t\left[\dot{\xi}+\frac{1}{2} \dot{x}_{i} \dot{x}_{i}\right] \text {. }
$$

which is the Lagrangian of a non-relativistic massive particle. This is invariant under the Schrödinger transformations (2.10). Equivalently, it shows the invariance up to total derivative under the Schrödinger symmetry of the Lagrangian $L=\frac{m}{2} \dot{x}_{i}^{2}$.

Let us construct the Hamiltonian for the Lagrangian (A.4). We calculate the conjugate momentum for $x_{i}$ and $v_{i}$ via

$$
p_{i}=\frac{\partial L}{\partial \dot{x}_{i}}=-\theta \epsilon_{i j} \dot{v}_{j}, \quad \pi_{i}=\frac{\partial L}{\partial \dot{v}_{i}}=\theta \epsilon_{i j}\left(\dot{x}_{j}+\frac{1}{2} v_{j}\right)
$$

and find

$$
H=p_{i} \dot{x}_{i}+\pi_{i} \dot{v}_{i}-L=-\frac{1}{\theta} \epsilon_{i j}\left(\pi_{j}-\frac{\theta}{2} \epsilon_{j k} v_{k}\right) p_{j} .
$$

Quantization proceeds by the replacement

$$
p_{i} \rightarrow-i \frac{\partial}{\partial x_{i}}, \quad \pi_{i} \rightarrow-i \frac{\partial}{\partial v_{i}} .
$$

Then the corresponding wave equation is

$$
\frac{\partial}{\partial t} \psi=-i H \psi=-\frac{1}{i \theta} \epsilon_{i j}\left(\frac{\partial}{\partial v_{i}}-\frac{i \theta}{2} \epsilon_{j k} v_{k}\right) \frac{\partial}{\partial x_{j}} \psi .
$$

This is exactly the free wave equation (3.12) discussed in the main text. 


\section{References}

[1] D. T. Son, "Toward an AdS/Cold Atoms Correspondence: a Geometric Realization of the Schrödinger Symmetry," Phys. Rev. D 78 (2008) 046003 arXiv:0804.3972 [hep-th]].

[2] K. Balasubramanian and J. McGreevy, "Gravity Duals for Non-Relativistic CFTs," Phys. Rev. Lett. 101 (2008) 061601 [arXiv:0804.4053 [hep-th]].

[3] Y. Nishida and D. T. Son, "Nonrelativistic Conformal Field Theories," Phys. Rev. D 76 (2007) 086004 arXiv:0706.3746 [hep-th]].

[4] J. Maldacena, D. Martelli and Y. Tachikawa, "Comments on String Theory Backgrounds with Non-Relativistic Conformal Symmetry," JHEP 0810 (2008) 072 arXiv:0807.1100 [hep-th]].

[5] C. P. Herzog, M. Rangamani and S. F. Ross, "Heating Up Galilean Holography," JHEP 0811 (2008) 080 [arXiv:0807.1099 [hep-th]].

[6] A. Adams, K. Balasubramanian and J. McGreevy, "Hot Spacetimes for Cold Atoms," JHEP 0811 (2008) 059 [arXiv:0807.1111 [hep-th]].

[7] G. Burdet, M. Perrin and P. Sorba, "About the Non-Relativistic Structure of the Conformal Algebra," Commun. Math. Phys. 34 (1973) 85.

[8] C. Duval, G. Burdet, H. P. Kunzle and M. Perrin, "Bargmann Structures and Newton-Cartan Theory," Phys. Rev. D 31 (1985) 1841.

[9] C. Duval, G. W. Gibbons and P. Horváthy, "Celestial Mechanics, Conformal Structures, and Gravitational Waves," Phys. Rev. D 43 (1991) 3907 arXiv:hep-th/0512188.

[10] A. Bergman and O. J. Ganor, "Dipoles, Twists and Noncommutative Gauge Theory," JHEP 0010 (2000) 018 arXiv:hep-th/0008030.

[11] S. Kachru, X. Liu and M. Mulligan, "Gravity Duals of Lifshitz-Like Fixed Points," Phys. Rev. D 78 (2008) 106005 [arXiv:0808.1725 [hep-th]].

[12] J. Negro, M. A. del Olmo and A. Rodríguez-Marco, "Nonrelativistic Conformal Groups. I," J. Math. Phys. 38 (1997) 3786. 
[13] J. Negro, M. A. del Olmo and A. Rodríguez-Marco, "Nonrelativistic Conformal Groups. II. Further Developments and Physical Applications," J. Math. Phys. 38 (1997) 3810 .

[14] C. Duval and P. A. Horváthy, "The exotic Galilei group and the 'Peierls substitution'," Phys. Lett. B 479, 284 (2000) arXiv:hep-th/0002233.

[15] S. Bhattacharyya, S. Minwalla and S. R. Wadia, "The Incompressible NonRelativistic Navier-Stokes Equation from Gravity," arXiv:0810.1545 [hep-th].

[16] I. Fouxon and Y. Oz, "CFT Hydrodynamics: Symmetries, Exact Solutions and Gravity," JHEP 0903 (2009) 120 [arXiv:0812.1266 [hep-th]].

[17] P. A. Horváthy, "Non-Commutative Mechanics, in Mathematical \& in Condensed Matter Physics," arXiv:cond-mat/0609571.

[18] P. C. Stichel and W. J. Zakrzewski, "A New Type of Conformal Dynamics," Annals Phys. 310 (2004) 158 arXiv:hep-th/0309038.

[19] J. Lukierski, P. C. Stichel and W. J. Zakrzewski, "Acceleration-Extended Galilean Symmetries with Central Charges and Their Dynamical Realizations," Phys. Lett. B 650 (2007) 203 arXiv:hep-th/0702179.

[20] C. Duval, M. Hassaine and P. A. Horváthy, "The Geometry of Schrödinger Symmetry in Gravity Background/Non-Relativistic CFT," Annals Phys. 324 (2009) 1158 [arXiv:0809.3128 [hep-th]].

[21] V. Bargmann, "On Unitary Ray Representations of Continuous Groups," Annals Math. 59 (1954) 1.

[22] E. İnönü and E. P. Wigner, "On the Contraction of Groups and Their Represenations," Proc. Nat. Acad. Sci. 39 (1953) 510.

[23] R. Jackiw and V. P. Nair, "Anyon Spin and the Exotic Central Extension of the Planar Galilei Group," Phys. Lett. B 480 (2000) 237 arXiv:hep-th/0003130.

[24] R. Jackiw and V. P. Nair, "Remarks on the Exotic Central Extension of the Planar Galilei Group," Phys. Lett. B 551 (2003) 166 arXiv:hep-th/0211119.

[25] C. Duval and P. A. Horváthy, "Spin and Exotic Galilean Symmetry," Phys. Lett. B 547 (2002) 306 [Erratum-ibid. B 588 (2004) 228] arXiv:hep-th/0209166]. 
[26] J. Lukierski, P. C. Stichel and W. J. Zakrzewski, "Exotic Galilean Conformal Symmetry and Its Dynamical Realisations," Phys. Lett. A 357 (2006) 1 arXiv:hep-th/0511259.

[27] D. E. Berenstein, J. M. Maldacena and H. S. Nastase, "Strings in Flat Space and PP Waves from $\mathcal{N}=4$ Super Yang Mills," JHEP 0204 (2002) 013 arXiv:hep-th/0202021.

[28] M. Kruczenski and A. A. Tseytlin, "Spiky Strings, Light-Like Wilson Loops and PP-Wave Anomaly," Phys. Rev. D 77 (2008) 126005 [arXiv:0802.2039 [hep-th]].

[29] P. A. Horváthy and M. S. Plyushchay, "Anyon Wave Equations and the Noncommutative Plane," Phys. Lett. B 595 (2004) 547 arXiv:hep-th/0404137].

[30] A. Bagchi and R. Gopakumar, "Galilean Conformal Algebras and AdS/CFT," arXiv:0902.1385 [hep-th].

[31] M. Alishahiha, A. Davody and A. Vahedi, "On AdS/CFT of Galilean Conformal Field Theories," arXiv:0903.3953 [hep-th].

[32] A. Bagchi and I. Mandal, "On Representations and Correlation Functions of Galilean Conformal Algebras," arXiv:0903.4524 [hep-th].

[33] M. Henkel, "Schrödinger Invariance in Strongly Anisotropic Critical Systems," J. Statist. Phys. 75 (1994) 1023 arXiv:hep-th/9310081].

[34] M. Henkel and J. Unterberger, "Schrödinger Invariance and Space-Time Symmetries," Nucl. Phys. B 660 (2003) 407 arXiv:hep-th/0302187.

[35] M. Alishahiha, R. Fareghbal, A. E. Mosaffa and S. Rouhani, "Asymptotic Symmetry of Geometries with Schrödinger Isometry," arXiv:0902.3916 [hep-th].

[36] J. D. Brown and M. Henneaux, "Central Charges in the Canonical Realization of Asymptotic Symmetries: an Example from Three-Dimensional Gravity," Commun. Math. Phys. 104 (1986) 207.

[37] J. M. Lévy-Leblond, "Galilei Group and Galilean Invariance," pp. 221-299 in "Group Theory and Its Applications, Vol. 2", (Ed. E. Loebl) Academic Press, New York, 1971. 
[38] D. R. Grigore, "The Projective Unitary Irreducible Representations of the Galilei Group in (1+2)-Dimensions," J. Math. Phys. 37 (1996) 460 arXiv:hep-th/9312048.

[39] S. K. Bose, "The Galilean Group in (2+1) Space-Times and Its Central Extension," Commun. Math. Phys. 169 (1995) 385.

[40] J. Lukierski, P. C. Stichel and W. J. Zakrzewski, "Galilean-Invariant (2+1)Dimensional Models with a Chern-Simons-Like Term And D $=2$ Noncommutative Geometry," Annals Phys. 260 (1997) 224 [arXiv:hep-th/9612017].

[41] M. A. del Olmo and M. S. Plyushchay, "Electric Chern-Simons Term, Enlarged Exotic Galilei Symmetry and Noncommutative Plane," Annals Phys. 321 (2006) 2830 arXiv:hep-th/0508020.

[42] J.-M. Lévy-Leblond, "Nonrelativistic Particles and Wave Equations," Comm. Math. Phys. 6, 4 (1967), 286-311.

[43] Y. Nakayama, "Index for Non-Relativistic Superconformal Field Theories," JHEP 0810 (2008) 083 [arXiv:0807.3344 [hep-th]].

[44] K. M. Lee, S. Lee and S. Lee, "Nonrelativistic Superconformal M2-Brane Theory," arXiv:0902.3857 [hep-th].

[45] A. Volovich and C. Wen, "Correlation Functions in Non-Relativistic Holography," arXiv:0903.2455 [hep-th].

[46] C. A. Fuertes and S. Moroz, "Correlation Functions in the Non-Relativistic AdS/CFT Correspondence," arXiv:0903.1844 [hep-th].

[47] S. S. Gubser, I. R. Klebanov and A. M. Polyakov, "Gauge Theory Correlators from Non-Critical String Theory," Phys. Lett. B 428 (1998) 105 arXiv:hep-th/9802109].

[48] E. Witten, "Anti-de Sitter Space and Holography," Adv. Theor. Math. Phys. 2 (1998) 253 arXiv:hep-th/9802150].

[49] O. Aharony, O. Bergman, D. L. Jafferis and J. Maldacena, "N $\mathcal{N}=6$ Superconformal Chern-Simons-Matter Theories, M2-Branes and Their Gravity Duals," JHEP 0810 (2008) 091 [arXiv:0806.1218 [hep-th]]. 